UCRL-53705

Distribution Category UC-2

\title{
DISCLAIMER
}

This report was prepared as an account of work sponsored by an agency of the United States Government. Neither the United States Government nor any agency thereof, nor any of their employees, makes any warranty, express or implied, or assumes any legal liability or responsibility for the accuracy, completeness, or usefulness of any information, apparatus, product, or process disclosed, or represents that its use would not infringe privately owned rights. Reference herein to any specific commercial product, process, or service by trade name, trademark, manufacturer, or otherwise does not necessarily constitute or imply its endorsement, recommendation, or favoring by the United States Government or any agency thereof. The views and opinions of authors expressed herein do not necessarily state or reflect those of the United States Government or any agency thereof.

\section{Results of Calculations of External Gamma Radiation Exposure Rates from Fallout and the Related Radionuclide Compositions-the Trinity Event}

\author{
H. G. Hicks
}

Manuscript date: December 1985

This document is

\section{PUBLICLY RELEASABLE}

Bany steel.

Authorizing Official

Date:

$r \cdot 24-42$

\section{LAWRENCE LIVERMORE NATIONAL LABORATORY University of California - Livermore, California • 94550}

Available from: National Technical Information Service • U.S. Department of Comm

5285 Port Royal Road - Springfield, VA 22161 - A02 - (Microfiche A01) \& 


\section{DISCLAIMER}

This report was prepared as an account of work sponsored by an agency of the United States Government. Neither the United States Government nor any agency Thereof, nor any of their employees, makes any warranty, express or implied, or assumes any legal liability or responsibility for the accuracy, completeness, or usefulness of any information, apparatus, product, or process disclosed, or represents that its use would not infringe privately owned rights. Reference herein to any specific commercial product, process, or service by trade name, trademark, manufacturer, or otherwise does not necessarily constitute or imply its endorsement, recommendation, or favoring by the United States Government or any agency thereof. The views and opinions of authors expressed herein do not necessarily state or reflect those of the United States Government or any agency thereof. 


\section{DISCLAIMER}

Portions of this document may be illegible in electronic image products. Images are produced from the best available original document. 


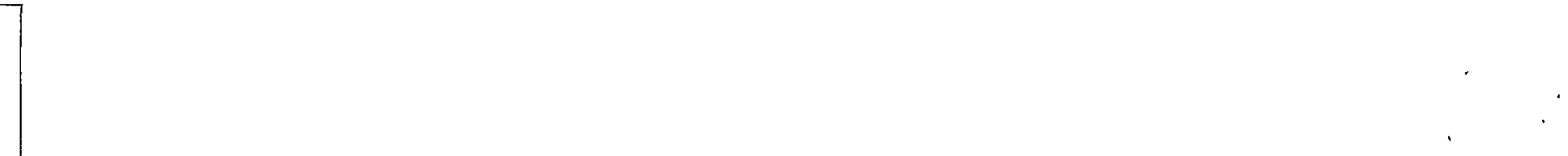




\section{Contents}

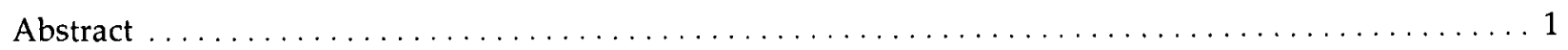

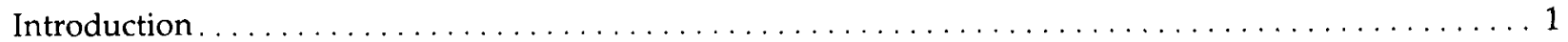

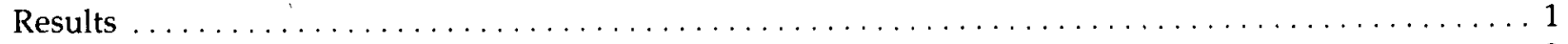

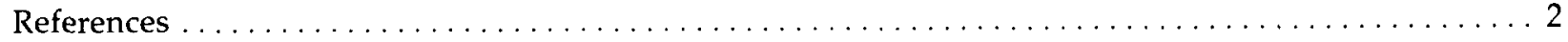

Appendix: Detailed Results for the Trinity Event $\ldots \ldots \ldots \ldots \ldots \ldots \ldots \ldots \ldots \ldots \ldots \ldots$ 


\title{
Results of Calculations of \\ External Gamma Radiation \\ Exposure Rates from Fallout \\ and the Related Radionuclide \\ Compositions-the Trinity \\ Event
}

\begin{abstract}
This report presents data on calculated gamma radiation exposure rates and ground deposition of related radionuclides resulting from the Trinity Event.
\end{abstract}

\section{Introduction}

In the past, we calculated the normalized external gamma radiation exposure rates resulting from local fallout from the Nevada Test Site (NTS) ${ }^{1}$ and from Pacific Tests. ${ }^{2}$ These calculations were used to reconstruct radiation doses downwind. Recently, we were asked to make the calculations for the 20-kt Trinity Event, fired on July 16, 1945 at Alamogordo, New Mexico.

\section{Results}

Results of the calculations of relative external gamma radiation exposure rates and related radionuclide ground deposition are given in the Appendix. Activation products created by neutron interaction with the earth and other environmental materials present on the earth's surface are unimportant in the context of food and water contamination and are therefore not included. Plutonium and most uranium isotopes are not included in the calculations because they do not contribute significantly to the external gamma radiation and to ensure that this document is not classified. These calculations are described in detail in Ref. 3. The output of the calculation has 30 decay times: 10 from 1 to 21 hours, 10 from 1 to 300 days, and 10 from 1 to 50 years. For each of these times and for zero time, there are values of the external gamma radiation exposure rate normalized to $1 \mathrm{mR} / \mathrm{h}$, 1 meter above the surface, 12 hours after the event; the associated values of $\mu \mathrm{Ci} / \mathrm{m}^{2}$ for each radionuclide; and the total $\mu \mathrm{Ci} / \mathrm{m}^{2}$.

Surface roughness effects were simulated by using Beck's values ${ }^{4}$ of $\left.(\mathrm{mR} / \mathrm{h}) / \mu \mathrm{Ci} / \mathrm{m}^{2}\right)$ for a re- laxation length of $0.16 \mathrm{~g} / \mathrm{cm}^{2}$. According to Beck, the concentration of fallout varies exponentially with soil depth $Z$, according to the relation $C=$ $C_{0} e^{-\alpha Z}$. He defines relaxation length as $1 / \alpha$.

Fractionation effects were simulated by the removal of one-half of the refractory nuclides from the calculation.

The Appendix contains 11 pages of calculated results that are marked Page 2 through Page 12. Page 2 gives the external gamma-ray exposure rates and associated values of total microcuries per square meter at 30 decay intervals and at zero time. The remaining pages give calculated values for each radionuclide at various decay intervalsfrom 1 to 21 hours on pp. 3-7, from 1 to 300 days on pp. 8-11, and from 1 to 50 years on p. 12. The production of nuclides designated by $\left({ }^{*}\right)$ has been estimated. When no estimate could be made, the value appears as zero. Note that the totals at zero time include orrly the listed nuclides and not all nuclides present at zero time. 


\section{References}

1. H. G. Hicks, Results of Calculations of External Gamma Radiation Exposure Rates from Fallout and the Related Radionuclide Compositions, Lawrence Livermore National Laboratory, Livermore, CA, UCRL53152 (1981), Parts 1-8.

Part 1-Operation Ranger, 1951

Part 2--Operation Buster-Jangle, 1951

Part 3-Operation Tumbler-Snapper, 1952

Part 4-Operation Upshot-Knothole, 1953

Part 5-Operation Teapot, 1955

Part 6-Operation Plumbob, 1957

Part 7-Operation Hardtack II, 1958

Part 8-Operations Nougat through Bowline, 1962-1968.

2. H. G. Hicks, Results of Calculations of External Gamma Radiation Exposure Rates from Local Fallout and the Related Radionuclide Compositions of Selected U.S. Pacific Events, Lawrence Livermore National Laboratory, Livermore, CA, UCRL-53505 (February 1984).

3. H. G. Hicks, "Calculation of the Concentration of Any Radionuclide Deposited on the Ground by Fallout from a Nuclear Detonation," Health Physics 42(5), 585-600 (May 1982).

4. H. L. Beck, Exposure Rate Conversion Factors for Radionuclides Deposited on the Ground, Environmental Measurements Laboratory, Department of Energy, New York, NY, EML-387 (July 1980).

$\mathrm{JCC} / \mathrm{djm}$ 
Appendix

Detailed Results for the Trinity Event 
TR IN ITY

MR/HR AT $\mathrm{H}+12$ HOURS $=1.000$

FRACTION OF REFRACTORIES PRESENT $=\varnothing .5 \varnothing \varnothing$

RELAXATION LENGTH $=\varnothing .16$ GM/SQCM
BOMB FRACTION PER SO. METER = 2.937E-13

TIME

ZERO TIME HOURS

$1 . \varnothing \varnothing E+\varnothing \varnothing$
$2 . \varnothing \varnothing E+\varnothing \varnothing$
$3 . \varnothing \varnothing E+\varnothing \varnothing$
$4 . \varnothing \varnothing E+\varnothing \varnothing$
$6 . \varnothing \varnothing E+\varnothing \varnothing$
$9 . \varnothing \varnothing E+\varnothing \varnothing$
$1.2 \varnothing E+\varnothing 1$
$1.5 \varnothing E+\varnothing 1$
$1.8 \varnothing E+\varnothing 1$
$2.1 \varnothing E+\varnothing 1$
$1 . \varnothing \varnothing E+\varnothing \varnothing$ DAYS
$2 . \varnothing \varnothing E+\varnothing \varnothing$
$5 . \varnothing \varnothing E+\varnothing \varnothing$
$1 . \varnothing \varnothing E+\varnothing 1$
$2 . \varnothing \varnothing E+\varnothing 1$
$3 . \varnothing \varnothing E+\varnothing 1$
$5 . \varnothing \varnothing E+\varnothing 1$
$1 . \varnothing \varnothing E+\varnothing 2$
$2 . \varnothing \varnothing E+\varnothing 2$
$3 . \varnothing \varnothing E+\varnothing 2$
$1 . \varnothing \varnothing E+\varnothing \varnothing ~ Y E A R S$
$1.5 \varnothing E+\varnothing \varnothing$
$2 . \varnothing \varnothing E+\varnothing \varnothing$
$3.5 \varnothing E+\varnothing \varnothing$
$5 . \varnothing \varnothing E+\varnothing \varnothing$
$7 . \varnothing \varnothing E+\varnothing \varnothing$
$1 . \varnothing \varnothing E+\varnothing 1$
$2 . \varnothing \varnothing E+\varnothing 1$
$3.5 \varnothing E+\varnothing 1$
$5 . \varnothing \varnothing E+\varnothing 1$

$M R / H R$

1. $22 \mathrm{E}+\varnothing 2$

$3.59 E+\varnothing 1$

1. $35 \mathrm{E}+\not 1$

$7.15 \mathrm{E}+\varnothing \varnothing$

4. $42 \mathrm{E}+\varnothing .8$

2. $39 \mathrm{E}+\varnothing 0$

1. $36 E+\varnothing .9$

1. $\varnothing \not E+\varnothing 0$

$7.91 \mathrm{E}-\varnothing 1$

$6.51 \mathrm{E}-\varnothing 1$

$5.49 E-D 1$

4. $67 E-\$ 1$

$2.25 \mathrm{E}-\varnothing 1$

$9.17 \mathrm{E}-\varnothing 2$

$4.11 \mathrm{E}-62$

1. $59 \mathrm{E}-02$

$9.1 \varnothing \mathrm{E}-\not 3$

$4.25 \mathrm{E}-83$

1. $47 \mathrm{E}-83$

$4.86 E-04$

$1.98 \mathrm{E}-04$

$5.600 \mathrm{E}-85$

3.31E- -05

1. $84 \mathrm{E}-05$

$1.32 E-85$

1. $\triangle 4 \mathrm{E}-\not 15$

$8.73 E-\varnothing 6$

$6.17 \mathrm{E}-106$

$4.21 \mathrm{E}-06$

2. $95 E-06$
MICROCURIES/SO METER

$$
\begin{aligned}
& 1.51 E+\varnothing 4 \\
& 2.84 E+\varnothing 3 \\
& 9.77 E+\varnothing 2 \\
& 5.55 E+\varnothing 2 \\
& 3.99 E+\varnothing 2 \\
& 2.7 \varnothing E+\varnothing 2 \\
& 1.9 \varnothing E+\varnothing 2 \\
& 1.49 E+\varnothing 2 \\
& 1.23 E+\varnothing 2 \\
& 1.95 E+\varnothing 2 \\
& 9.11 E+\varnothing 1 \\
& 7.98 E+\varnothing 1 \\
& 4.17 E+\varnothing 1 \\
& 1.64 E+\varnothing 1 \\
& 6.43 E+\varnothing \varnothing \\
& 2.29 E+\varnothing \varnothing \\
& 1.34 E+\varnothing \varnothing 1 \\
& 7.22 E-\varnothing 1 \\
& 3 . \varnothing 9 E-\varnothing 1 \\
& 1.1 \varnothing E-\varnothing 1 \\
& 5.76 E-\varnothing 2 \\
& 4.37 E-\varnothing 2 \\
& 2.67 E-\varnothing 2 \\
& 1.9 \varnothing E-\varnothing 2 \\
& 8.67 E-\varnothing 3 \\
& 5 . \varnothing 9 E-\varnothing 3 \\
& 3.45 E-\varnothing 3 \\
& 2.69 E-\varnothing 3 \\
& 1.89 E-\varnothing 3 \\
& 1.3 \varnothing E-\varnothing 3 \\
& 9 . \varnothing 7 E-\varnothing 4 \\
&
\end{aligned}
$$


FRACTION OF REFRACTORIES PRESENT $=\varnothing .5 \varnothing 0$

RELAXATION LENGTH $=\varnothing .16 \mathrm{GM} / \mathrm{SOCM}$

BOMB FRACTION PER SO. METER = 2.937E-13

DEBRIS DECAY FROM 1 TO 21 HOURS

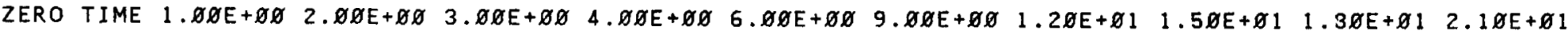

*BE 7

*NA 24

$\star M N 54$

*FE 55

FE 59

CO 57

*CU 64

*CU 67

W1 81

W185

W187

AU198

AU198

ת $\mathrm{PB} 203$

U237

* 4239

-NP239

*NP2 $4 \varnothing M$

*NP24Ø

AM241

CM242

GE 75

GE 77

AS 77

SE $77 M$

GE 78

AS 78

AS 79

SE $79 M$

BR $8 \varnothing$

SE $81 M$

SE 81

BR 82

SE 83

BR 83

$\begin{array}{ll}\text { KR } & 83 \mathrm{M}\end{array}$

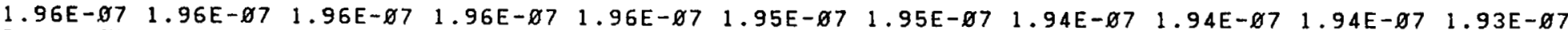

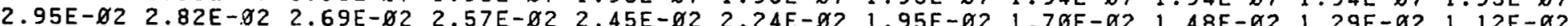

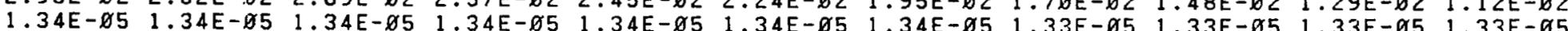

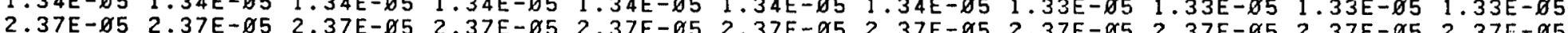

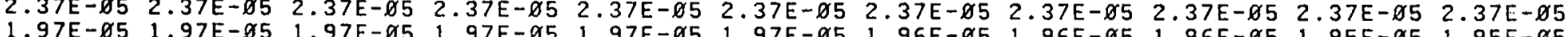

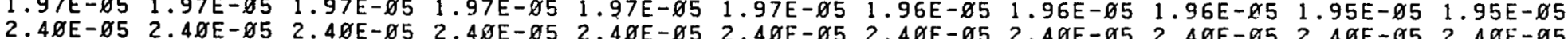

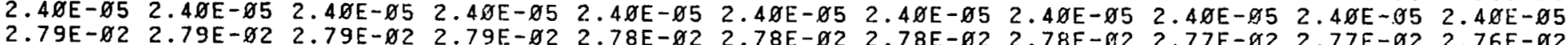

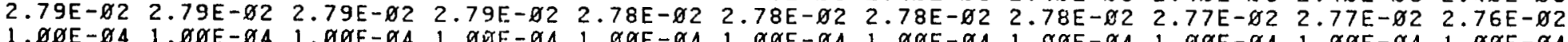

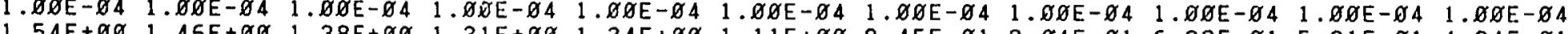

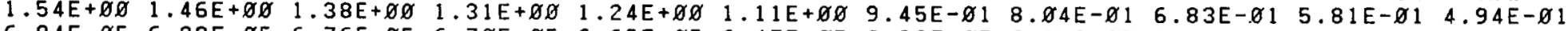
$6.94 \mathrm{E}-\varnothing 5 \quad 6.88 \mathrm{E}-\varnothing 5 \quad 6.76 \mathrm{E}-\varnothing 5 \quad 6.70 \mathrm{E}-\varnothing 5 \quad 6.65 \mathrm{E}-\varnothing 5 \quad 6.47 \mathrm{E}-\varnothing 5 \quad 6.23 \mathrm{E}-\varnothing 5 \quad 6.05 \mathrm{E}-\not 95 \quad 5.86 \mathrm{E}-\varnothing 5 \quad 5.64 \mathrm{E}-\varnothing 5 \quad 5.48 \mathrm{E}-\varnothing 5$

ס.

D.

. $\varnothing$.

Ø.

$\theta$.

$\varnothing$.

$\infty$.

$\varnothing . \quad \varnothing . \quad \varnothing$

$.6 \varnothing E+\varnothing \varnothing 159 E+\varnothing 01.59 E+\varnothing 001.58 E+\varnothing 001$

$\varnothing$

$\varnothing$

a.

$0.23 E-05$

0 .

.

$\varnothing$.

$\varnothing$.

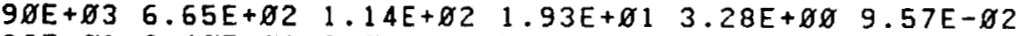

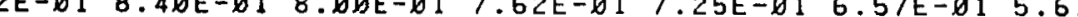

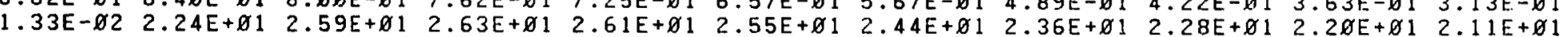

$\begin{array}{lllllllllll}0.00-\varnothing 3 & 8.44 E-\varnothing 1 & 8.07 E-\varnothing 1 & 7.69 E-\varnothing 1 & 7.32 E-\varnothing 1 & 6.62 E-\varnothing 1 & 5.72 E-\varnothing 1 & 4.94 E-\varnothing 1 & 4.25 E-\varnothing 1 & 3.67 E-\varnothing 1 & 3.17 E-\varnothing 1\end{array}$ $3.53 \mathrm{E}-111.82 \mathrm{E}-11 \mathrm{~g} .44 \mathrm{E}-12 \quad 4.87 \mathrm{E}-12 \quad 2.52 \mathrm{E}-126.74 \mathrm{E}-13$

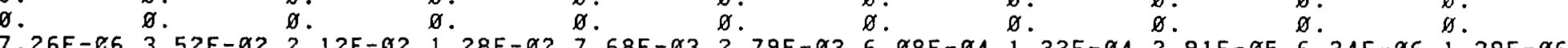

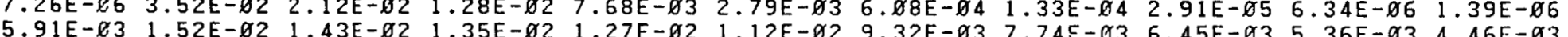
5.91E-03 $1.52 E-021.43 E-021.35 E-021.27 E-021.12 E-029.32 E-037.74 E-03-6.45 E-035.36 E$

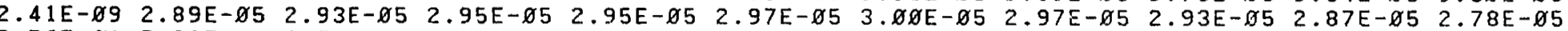

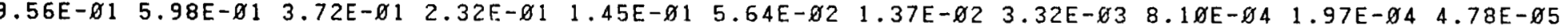

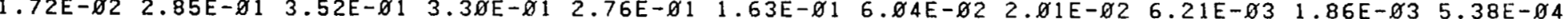

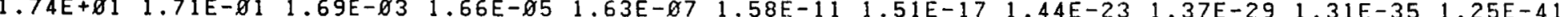

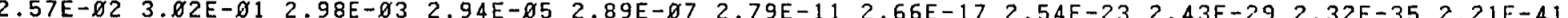

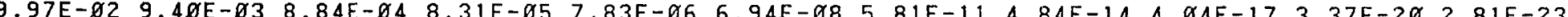

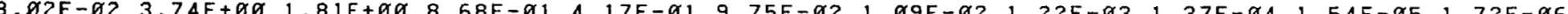

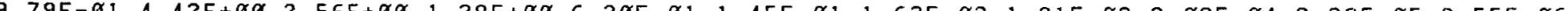

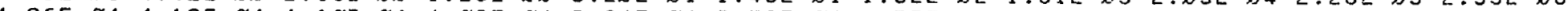

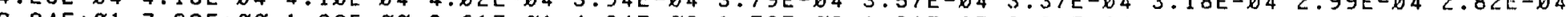

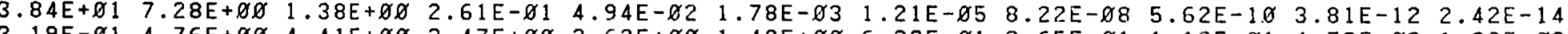

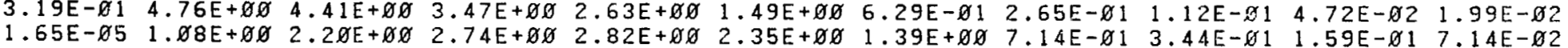


DEBRIS DECAY FROM 1 TO 21 HOURS

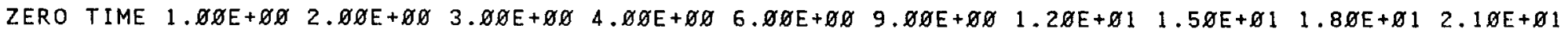

BR 84

KR $85 M$

$\begin{array}{ll}K R & 87\end{array}$

KR 88

RB 88

RB 89

SR 89

SR 90

SR 91

$Y 91 \mathrm{M}$

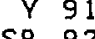

SR 92

$\begin{array}{rr}Y & 92 \\ \text { SR } & 93\end{array}$

$Y 93$

$Y 94$

- $Y 95$

ZR 95

NB 95

ZR 97

NB $97 \mathrm{M}$

NB $97 M$

NB 97

MO 99

TC $99 \mathrm{M}$

MO1ס 1

TC $1 \varnothing 1$

MO102

TC1 182M

TC182

RU1.03

RH103M

TC1 104

$\mathrm{RU} 185$

RH1Ø5M

RH 105

RU106

RHIØ6

RH 107

PD $107 M$

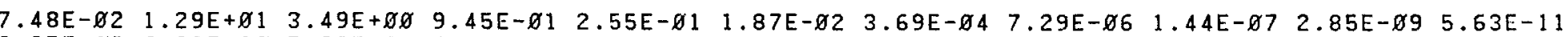

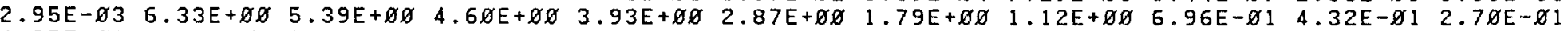

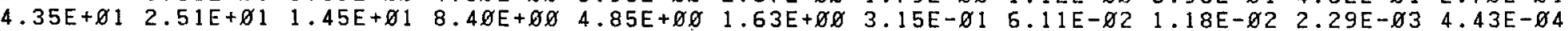

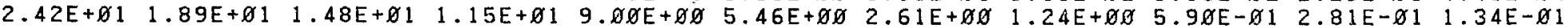

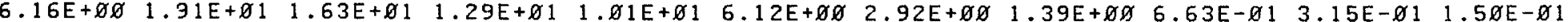
$3.2 \varnothing \mathrm{E}+\varnothing 1 \quad 3 . \varnothing 1 \mathrm{E}+\varnothing 1 \quad 2 . \varnothing 2 \mathrm{E}+\varnothing \varnothing 01.36 \mathrm{E}-\varnothing 1 \quad 9.11 \mathrm{E}-\varnothing 3 \quad 4.12 \mathrm{E}-\varnothing 5 \quad 1.25 \mathrm{E}-\varnothing 8 \quad 3.77 \mathrm{E}-12 \quad 1.15 \mathrm{E}-15 \quad 3.48 \mathrm{E}-19 \quad 1 . \varnothing 5 \mathrm{E}-22$

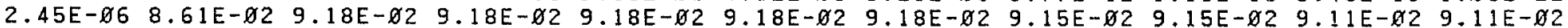

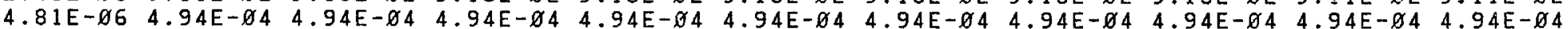

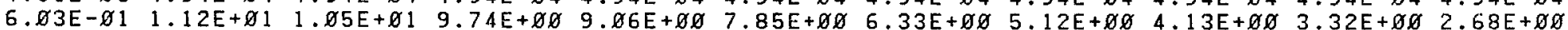

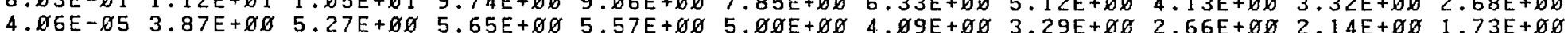

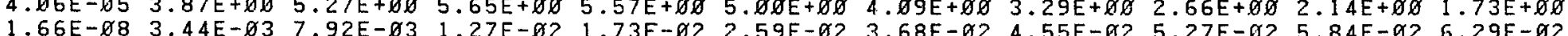

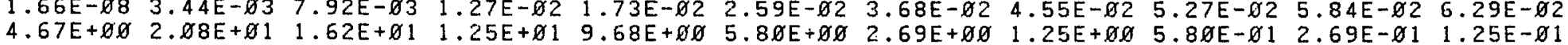

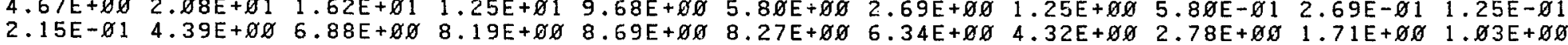

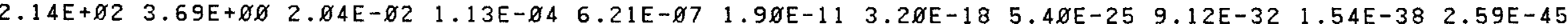
$2.62 \mathrm{E}-\varnothing 1 \quad 8.46 \mathrm{E}+\varnothing \varnothing 07.95 \mathrm{E}+\varnothing \varnothing \quad 7.43 \mathrm{E}+\varnothing \varnothing \varnothing \quad 6.95 \mathrm{E}+\varnothing \varnothing \quad 6 . \varnothing 6 \mathrm{E}+\varnothing \varnothing \quad 4.94 \mathrm{E}+\varnothing \varnothing \varnothing \quad 4 . \varnothing 4 \mathrm{E}+\varnothing \varnothing \quad 3.28 \mathrm{E}+\varnothing \varnothing \quad 2.69 \mathrm{E}+\varnothing \varnothing \quad 2.19 \mathrm{E}+\varnothing \varnothing$

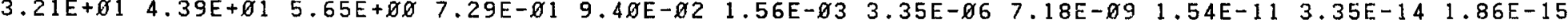

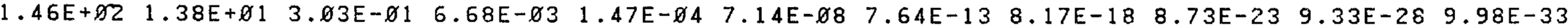

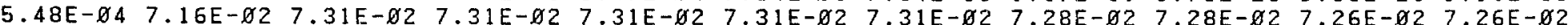

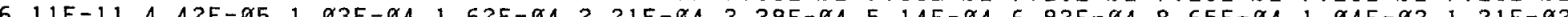

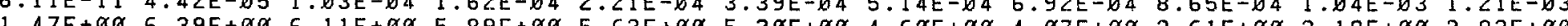
1.47E+ 7. $7.35 E-\varnothing 103.21 E+\varnothing \varnothing 04.52 E+\varnothing \varnothing \quad 5.15 E+\varnothing \varnothing \quad 5.41 E+\varnothing \varnothing \quad 5.38 E+\varnothing \varnothing \quad 4.9 \varnothing E+\varnothing \varnothing \quad 4.37 E+\varnothing \varnothing \quad 3.86 E+\varnothing \varnothing \quad 3.41 E+\varnothing \varnothing \quad 3 . \varnothing 3 E+\varnothing \varnothing$

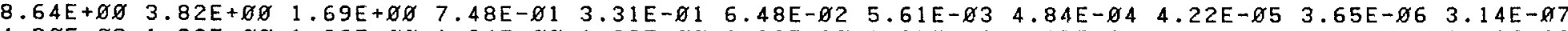

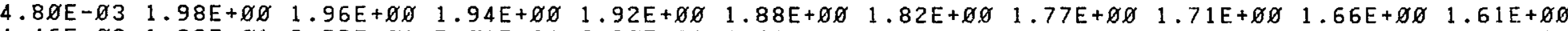

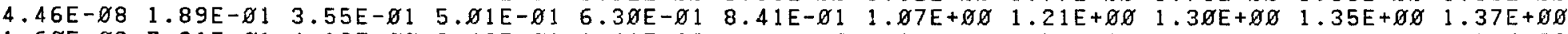
$\begin{array}{lllllllllll}1.6 \varnothing E+\varnothing 2 & 7.21 E+\varnothing 1 & 4.19 E+\varnothing \varnothing & 2.43 E-\varnothing 1 & 1.41 E-\varnothing 2 & 4.72 E-\varnothing 5 & 9.17 E-\varnothing 9 & 1.79 E-12 & 3.47 E-16 & 6.74 E-2 \varnothing 1.31 E-23\end{array}$

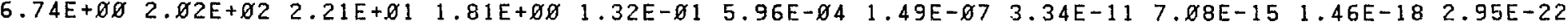
$1.52 \mathrm{E}+\varnothing 3 \quad 3.46 \mathrm{E}+\varnothing 1 \quad 7.88 \mathrm{E}-\varnothing 1 \quad 1.80 \mathrm{E}-\varnothing 2 \quad 4.11 \mathrm{E}-\varnothing 4 \quad 2.13 \mathrm{E}-\varnothing 7 \quad 2.54 \mathrm{E}-12 \quad 3.01 \mathrm{E}-17 \quad 3.57 \mathrm{E}-22 \quad 4.23 \mathrm{E}-27 \quad 5.03 \mathrm{E}-32$

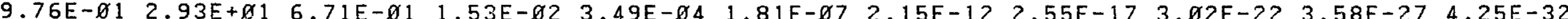

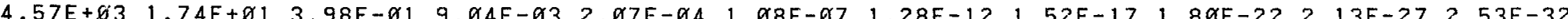

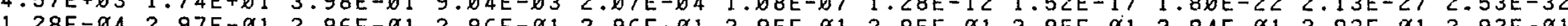

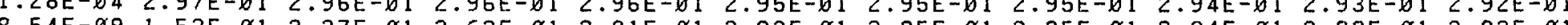
2. $11 E-012.92 E-012.95 E-012.95 E-012.94 E-012.93 E-\varnothing 12.93 E-\varnothing 1$ $1.19 E+\varnothing 28.56 E+\varnothing 18.56 E+\varnothing 08.48 E-\varnothing 18.38 E-\varnothing 28.26 E-\varnothing 4 \quad 8.06 E-\varnothing 7.7 .88 E-1007.69 E-137.52 E-167.34 \mathrm{E}-19$

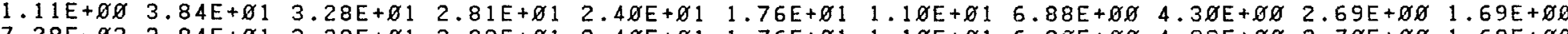
$\begin{array}{lllllllllll}7.29 E-\varnothing 3 & 3.84 E+\varnothing 1 & 3.29 E+\varnothing 1 & 2.82 E+\varnothing 1 & 2.4 \varnothing E+\varnothing 1 & 1.76 E+\varnothing 1 & 1.1 \varnothing E+\varnothing 1 & 6.9 \varnothing E+\varnothing \varnothing 0 & 4.32 E+\varnothing \varnothing & 2.7 \varnothing E+\varnothing \varnothing \quad 1.69 E+\varnothing \varnothing\end{array}$

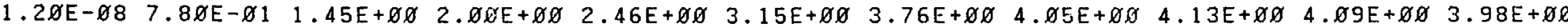

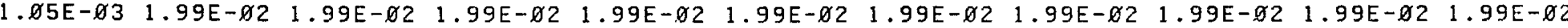

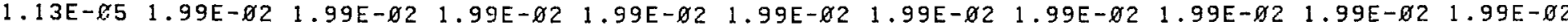

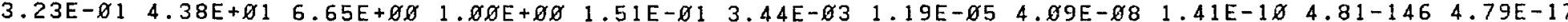
$6.71 \mathrm{E}-\varnothing 4 \quad 8.92 \mathrm{E}+\varnothing \varnothing \quad 1.35 \mathrm{E}+\varnothing \varnothing \quad 2 . \varnothing 4 \mathrm{E}-\varnothing 1 \quad 3 . \varnothing 7 \mathrm{E}-\varnothing 2 \quad 7 . \varnothing \varnothing \mathrm{E}-\varnothing 4 \quad 2.41 \mathrm{E}-\varnothing 6 \quad 8.32 \mathrm{E}-\varnothing 09 \quad 2.86 \mathrm{E}-1119.88 \mathrm{E}-14 \quad 3.37 \mathrm{E}-16$ 
DEBRIS DECAY FROM 1 TO 21 HOURS

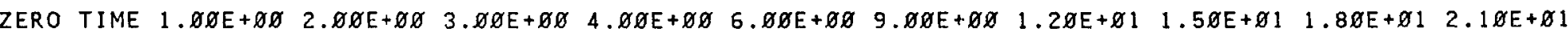

PD 109

$A G 109 M$

PDIIIM

PD 1111

AGI11M

AGI11

AG 112

$A G 113$

$A G 115$

CD115M

CDI15

I N $115 \mathrm{M}$

CD117

IN $117 \mathrm{M}$

IN 117

$\checkmark$ CD118

IN 118

CD 119

IN $119 \mathrm{M}$

INIII

SN 121

SN123M

SN 123

SN 125

SB 125

$S B 126$

SN 127

SB 127

TE127

SN128

SN 128

SB $128 M$

SB 128

SN129M

SN 129

SB 129

TE $129 \mathrm{M}$

TE 129

SB $130 \mathrm{M}$

SB $13 \varnothing$

$\begin{array}{lllllllllll}1.86 E-\varnothing 2 & 1.54 E+\varnothing \varnothing & 1.46 E+\varnothing \varnothing & 1.39 E+\varnothing \varnothing & 1.32 E+\varnothing \varnothing & 1.19 E+\varnothing \varnothing & 1 . \varnothing 2 E+\varnothing \varnothing & 8.77 E-\varnothing 1 & 7.51 E-\varnothing 1 & 6.44 E-\varnothing 1 & 5.53 E-\varnothing 1\end{array}$ $1 . \varnothing 7 E-\varnothing 4 \quad 1.54 E+\varnothing \varnothing \quad 1.46 E+\varnothing \varnothing \quad 1.39 E+\varnothing \varnothing \quad 1.32 E+\varnothing \varnothing \quad 1.19 E+\varnothing \varnothing \varnothing 1 . \varnothing 2 E+\varnothing \varnothing \quad 8.77 E-\varnothing 1 \quad 7.51 E-\varnothing 1 \quad 6.44 E-\varnothing 1 \quad 5.53 E-\varnothing 1$

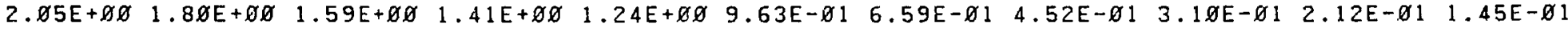
$\begin{array}{llllllllllll}7.29 E-\varnothing 1 & 1.31 \mathrm{E}+\varnothing \varnothing & 1.26 \mathrm{E}+\varnothing \varnothing & 1.12 \mathrm{E}+\varnothing \varnothing \varnothing & 9.93 \mathrm{E}-\varnothing 1 & 7.72 \mathrm{E}-\varnothing 1 & 5.3 \varnothing \mathrm{E}-\varnothing 1 & 3.63 \mathrm{E}-\varnothing 1 & 2.48 \mathrm{E}-\varnothing 1 & 1.7 \varnothing \mathrm{E}-\varnothing 1 & 1.17 \mathrm{E}-\varnothing 1\end{array}$

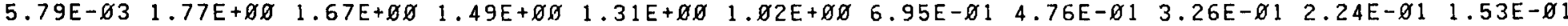

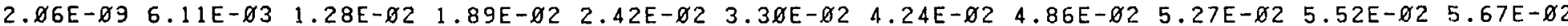

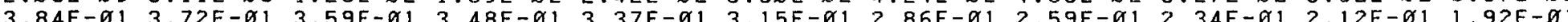
(12. $1.16 E-05$ 7.

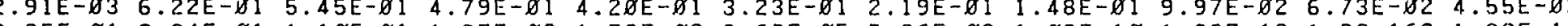

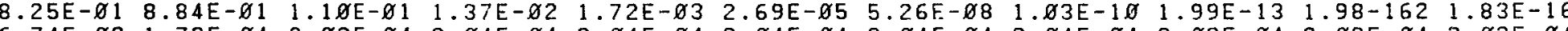

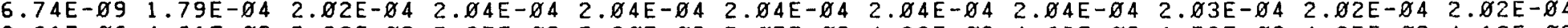

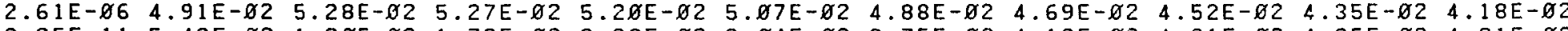
$3.25 \mathrm{E}-11 \quad 5.42 \mathrm{E}-\varnothing 3 \quad 1.2 \varnothing \mathrm{E}-\varnothing 2 \quad 1.79 \mathrm{E}-\varnothing 2 \quad 2.28 \mathrm{E}-\varnothing 2 \quad 3 . \varnothing 4 \mathrm{E}-\varnothing 2 \quad 3.75 \mathrm{E}-\varnothing 2 \quad 4.13 \mathrm{E}-\varnothing 2 \quad 4.31 \mathrm{E}-\varnothing 2 \quad 4.35 \mathrm{E}-\varnothing 2 \quad 4.31 \mathrm{E}-\varnothing 2$ $\begin{array}{lllllllllll}5.73 E-\varnothing 2 & 9.53 E-\varnothing 1 & 7.18 E-\varnothing 1 & 5.36 E-\varnothing 1 & 4.02 E-\varnothing 1 & 2.26 E-\varnothing 1 & 9.46 E-\varnothing 2 & 3.99 E-\varnothing 2 & 1.68 E-\varnothing 2 & 7.05 E-\not 03 & 2.97 E-\varnothing 3\end{array}$

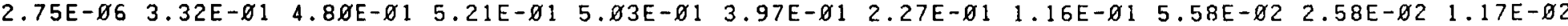

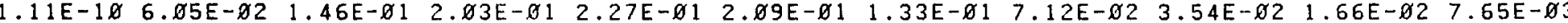

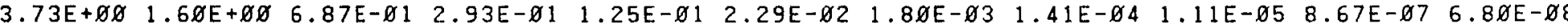

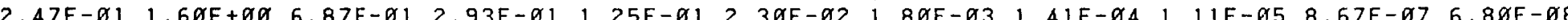

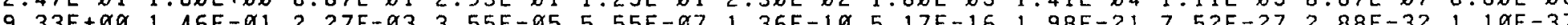
100

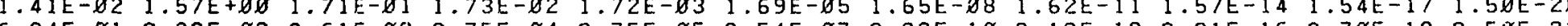
1.

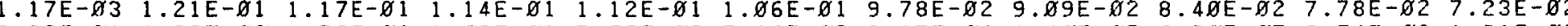
$6.92 E-\varnothing 1 \quad 1.29 E+\varnothing \varnothing 04.59 E-\varnothing 1 \quad 1.62 E-\varnothing 1 \quad 5.73 E-\varnothing 2 \quad 7.16 E-\varnothing 3 \quad 3.17 E-\varnothing 4 \quad 1.40 E-\varnothing 5 \quad 6.20 E-\varnothing 7 \quad 2.74 E-\varnothing 8 \quad 1.21 E-\varnothing 9$

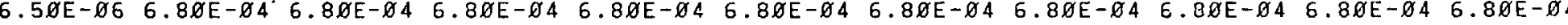

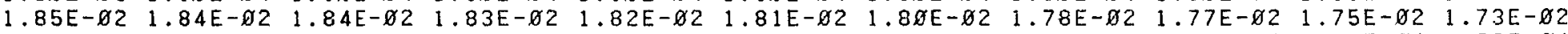

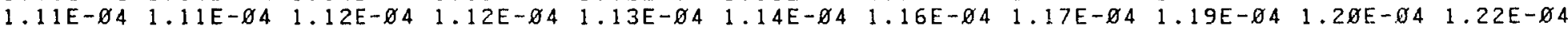

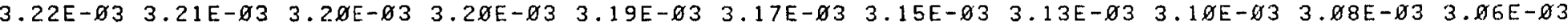

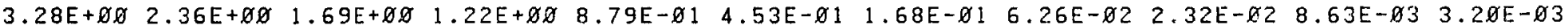

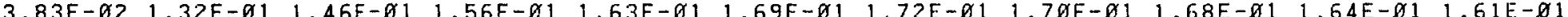

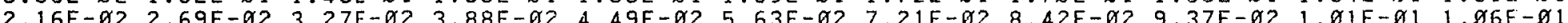

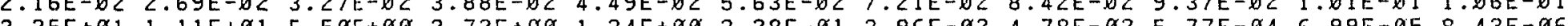

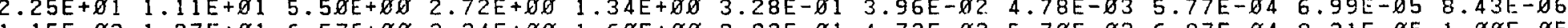

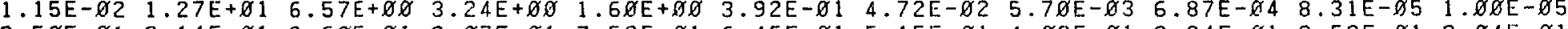

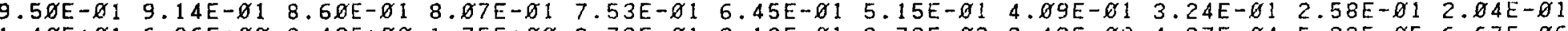

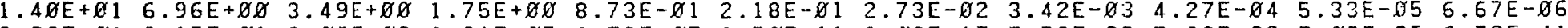

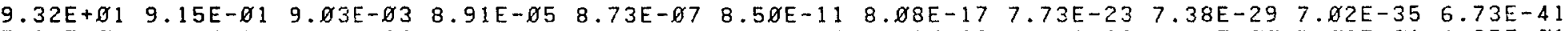

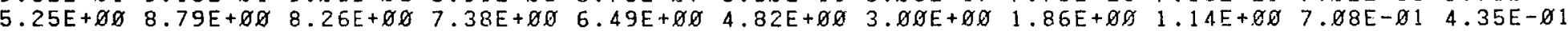

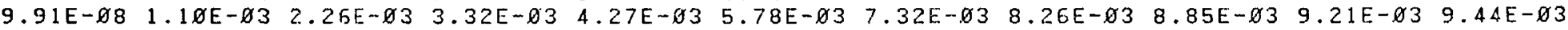
$3.78 \mathrm{E}+\varnothing \varnothing \quad 5.19 \mathrm{E}+\varnothing \varnothing \varnothing \quad 6 . \varnothing 8 \mathrm{E}+\varnothing \varnothing \quad 6.31 \mathrm{E}+\varnothing \varnothing \varnothing \quad 6 . \varnothing 8 \mathrm{E}+\varnothing \varnothing \varnothing 5 . \varnothing 3 \mathrm{E}+\varnothing \varnothing \quad 3.35 \mathrm{E}+\varnothing \varnothing 12.11 \mathrm{E}+\varnothing \varnothing 01.32 \mathrm{E}+\varnothing \varnothing \quad 8.14 \mathrm{E}-\varnothing 1 \quad 5 . \varnothing 5 \mathrm{E}-\varnothing 1$

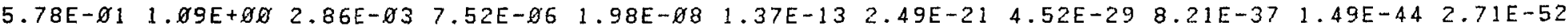

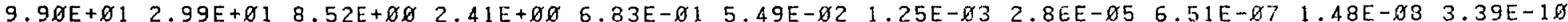




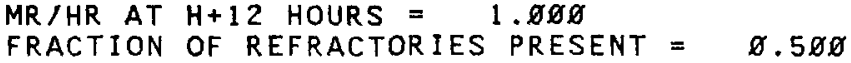

FRACTION OF REFRACTORIES PRESENT $=\varnothing .500$
RELAXATION LENGTH $=\varnothing .16 \mathrm{GM} / \mathrm{SOCM}$

RELAXATION LENGTH $=\varnothing .16 \mathrm{GM} / \mathrm{SOCM}$
BOMB FRACTION PER SQ. METER $=2.937 \mathrm{E}-13$

DEBRIS DECAY FROM 1 TO 21 HOURS

ZERO TIME $1 . \varnothing \varnothing E+\varnothing \varnothing \quad 2 . \varnothing \varnothing E+\varnothing \varnothing \quad 3 . \varnothing \varnothing E+\varnothing \varnothing \quad 4 . \varnothing \varnothing E+\varnothing \varnothing \quad 6 . \varnothing \varnothing E+\varnothing \varnothing \quad 9 . \varnothing \varnothing E+\varnothing \varnothing \quad 1.2 \varnothing E+\varnothing 1 \quad 1.5 \varnothing E+\varnothing 1 \quad 1.8 \varnothing E+\varnothing 1 \quad 2.1 \varnothing E+\varnothing 1$

I $13 \varnothing$

SB 131

TE $131 \mathrm{M}$

TE 13

I 131

TE 132

TE 1332

TE 133

I 133

XE133M

XE 133

TE 134

I 134

XE135M

$\infty$ XE135

CSI3

CS 137

XE 138

CS 138

$\operatorname{CS} 139$

BA 139

BA 140

A 140

BA141

BA141

CE141

BA 142

LA 142

LA 43

CE 143

PR 143

CE 144

PR 144

CE 145

CE 146

PR 146
PR 147

$2.76 \mathrm{E}-\varnothing 2 \quad 2.61 \mathrm{E}-\varnothing 2 \quad 2.47 \mathrm{E}-\varnothing 2 \quad 2.33 \mathrm{E}-\varnothing 2 \quad 2.2 \varnothing \mathrm{E}-\varnothing 2 \quad 1.97 \mathrm{E}-\varnothing 2 \quad 1.67 \mathrm{E}-\varnothing 2 \quad 1.41 \mathrm{E}-\varnothing 2 \quad 1.19 \mathrm{E}-\varnothing 2 \quad 1.01 \mathrm{E}-\varnothing 2 \quad 8.52 \mathrm{E}-\varnothing 3$

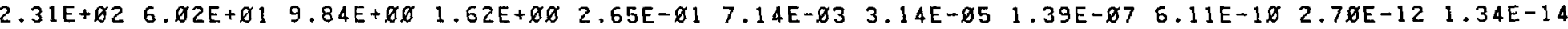

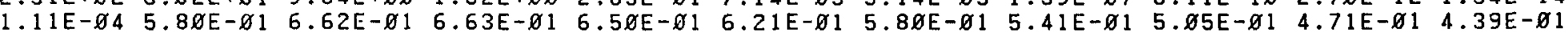

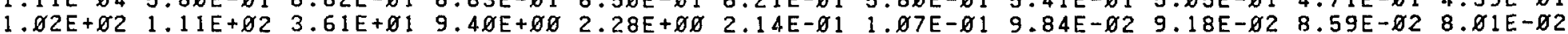

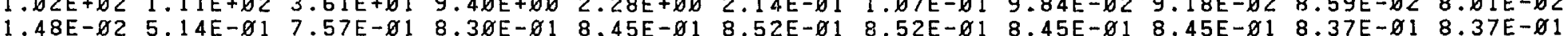

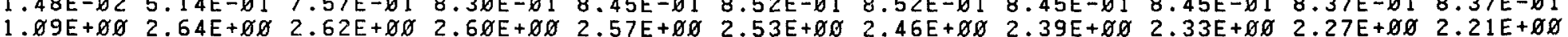

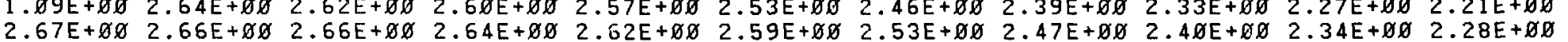

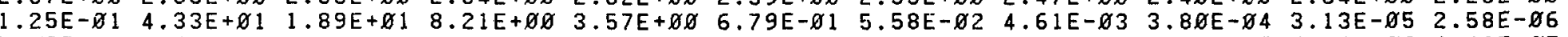

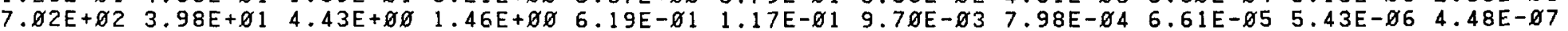

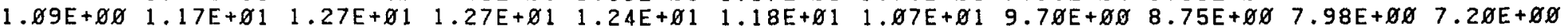

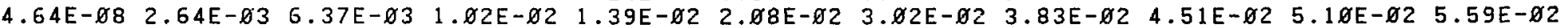

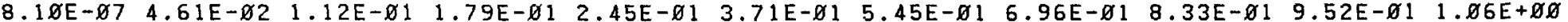
$2.33 E+\varnothing 2 \quad 1.08 E+02 \quad 4.02 E+\varnothing 1 \quad 1.49 E+\varnothing 1 \quad 5.54 E+\varnothing 007.69 E-01 \quad 3.93 E-02 \quad 2.01 E-03 \quad 1.03 E-04 \quad 5.29 E-062.71 E-07$

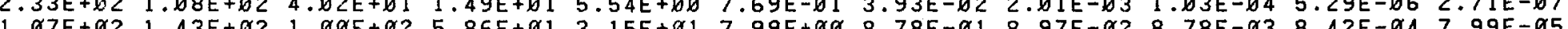

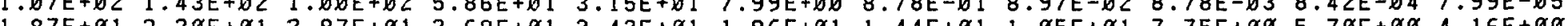
更

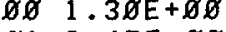

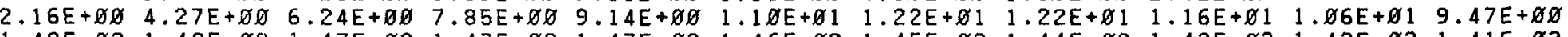

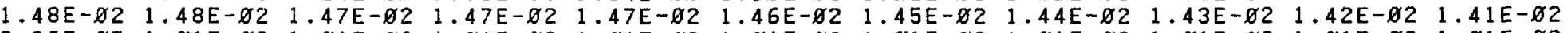
$9.36 \mathrm{E}-\varnothing 5 \quad 1.01 \mathrm{E}-\varnothing 3 \quad 1 . \varnothing 1 \mathrm{E}-\varnothing 3 \quad 1 . \varnothing 1 \mathrm{E}-\varnothing 3 \quad 1 . \varnothing 1 \mathrm{E}-\varnothing 3 \quad 1 . \varnothing 1 \mathrm{E}-\varnothing 3 \quad 1 . \varnothing 1 \mathrm{E}-\varnothing 3 \quad 1 . \varnothing 1 \mathrm{E}-\varnothing 3 \quad 1.01 \mathrm{E}-\varnothing 3 \quad 1 . \varnothing 1 \mathrm{E}-\varnothing 3 \quad 1 . \varnothing 1 \mathrm{E}-\varnothing 3$

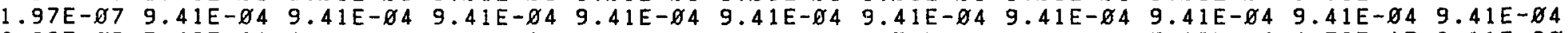
$6.38 \mathrm{E}+\varnothing 2 \quad 5.49 \mathrm{E}+\varnothing 1 \quad 4.78 \mathrm{E}+\varnothing \varnothing \quad 4.14 \mathrm{E}-\varnothing 1 \quad 3.58 \mathrm{E}-\varnothing 2 \quad 2.69 \mathrm{E}-\varnothing 4 \quad 1.75 \mathrm{E}-\varnothing 7 \quad 1.13 \mathrm{E}-1 \varnothing \quad 7.37 \mathrm{E}-14 \quad 4.79 \mathrm{E}-17 \quad 3.11 \mathrm{E}-2 \varnothing$

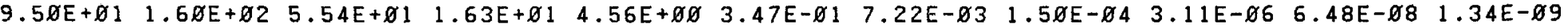

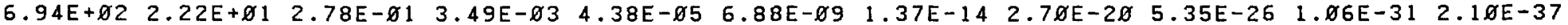
$\begin{array}{llllllllllll}1.28 E+\varnothing 1 & 1.43 E+\varnothing 2 & 8.81 E+\varnothing 1 & 5.35 E+\varnothing 1 & 3.24 E+\varnothing 1 & 1.19 E+\varnothing 1 & 2.64 E+\varnothing \varnothing 05.85 E-\varnothing 1 & 1.30 E-\varnothing 1 & 2.88 E-\varnothing 2 & 6.41 E-\varnothing 3\end{array}$

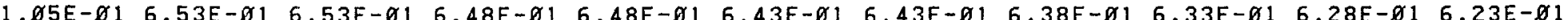
$2.49 E-071.12 E-022.21 E-02 \quad 3.29 E-02 \quad 4.34 E-020.38 E-02 \quad 9.32 E-02 \quad 1.21 E-011.47 E-011.71 E-011.94 E-01$ $1.45 E$

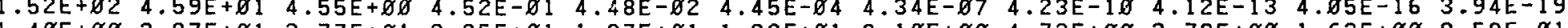

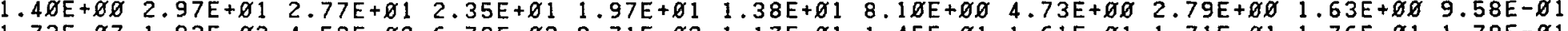

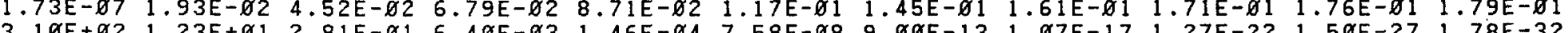
$3.1 \varnothing \mathrm{E}+\varnothing 21.23 \mathrm{E}+\varnothing 1 \quad 2.81 \mathrm{E}-\varnothing 1 \quad 6.4 \varnothing \mathrm{E}-\varnothing 3 \quad 1.46 \mathrm{E}-\varnothing 4 \quad 7.58 \mathrm{E}-\varnothing 8 \quad 9 . \varnothing \varnothing \mathrm{E}-13 \quad 1 . \varnothing 7 \mathrm{E}-17 \quad 1.27 \mathrm{E}-22 \quad 1.5 \varnothing \mathrm{E}-27 \quad 1.78 \mathrm{E}-32$ $\begin{array}{lllllllllll}7.11 \mathrm{E}+\varnothing \varnothing 0 & 4.93 \mathrm{E}+\varnothing 1 & 3.25 \mathrm{E}+\varnothing 1 & 2 . \varnothing 7 \mathrm{E}+\varnothing 1 & 1.32 \mathrm{E}+\varnothing 1 & 5.33 \mathrm{E}+\varnothing \varnothing & 1.37 \mathrm{E}+\varnothing \varnothing & 3.54 \mathrm{E}-\varnothing 1 & 9.13 \mathrm{E}-\varnothing 2 & 2.35 \mathrm{E}-\varnothing 2 & 6 . \varnothing 6 \mathrm{E}-\varnothing 3\end{array}$

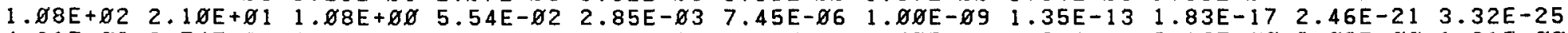

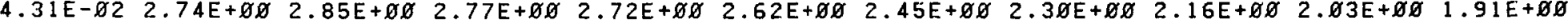

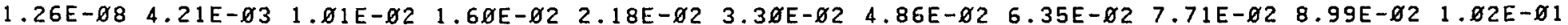
$\begin{array}{lllllllllll}1.72 \mathrm{E}-\varnothing 3 & 1.23 \mathrm{E}-\varnothing 2 & 1.23 \mathrm{E}-\varnothing 2 & 1.23 \mathrm{E}-\varnothing 2 & 1.23 \mathrm{E}-\varnothing 2 & 1.23 \mathrm{E}-\varnothing 2 & 1.23 \mathrm{E}-\varnothing 2 & 1.23 \mathrm{E}-\varnothing 2 & 1.23 \mathrm{E}-\varnothing 2 & 1.23 \mathrm{E}-\varnothing 2 & 1.23 \mathrm{E}-\varnothing 2\end{array}$

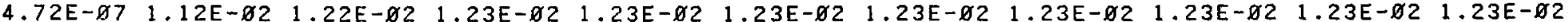

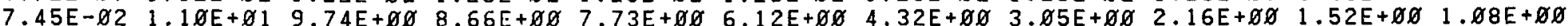

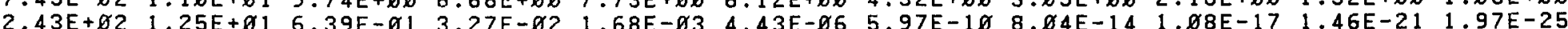
$5.43 E+02$ 1.25E+

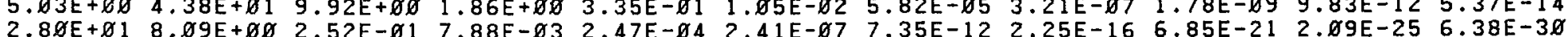


FRACTION OF REFRACTORIES PRESENT $=\varnothing .5 \varnothing \varnothing$

RELAXATION LENGTH $=\varnothing .16 \mathrm{GM} / \mathrm{SQCM}$

BOMB FRACTION PER SQ. METER $=2.937 E-13$

DEBRIS DECAY FROM 1 TO 21 HOURS

ZERO TIME $1 . \varnothing \varnothing E+\varnothing \varnothing \quad 2 . \varnothing \varnothing E+\varnothing \varnothing \quad 3 . \varnothing \varnothing E+\varnothing \varnothing \quad 4 . \varnothing \varnothing E+\varnothing \varnothing \quad 6 . \varnothing \varnothing E+\varnothing \varnothing \varnothing . \varnothing \varnothing E+\varnothing \varnothing \quad 1.2 \varnothing E+\varnothing 1 \quad 1.5 \varnothing E+\varnothing 1 \quad 1.8 \varnothing E+\varnothing 1 \quad 2.1 \varnothing E+\varnothing 1$

ND 147

PM15Ø

ND 151

PM151

PM152

SM153

SM 155

EUI55

SM156

EU156

EU 157

EU158

GD 159

o TB 161

TOTAL

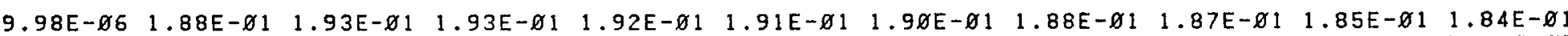

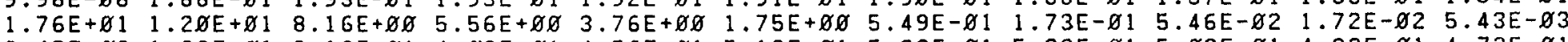

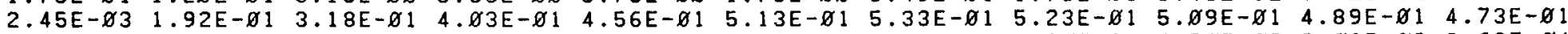

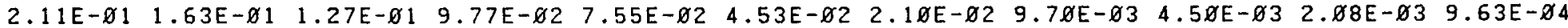
$8.36 \mathrm{E}+\varnothing 1 \quad 2.61 \mathrm{E}+\varnothing \varnothing \quad 8.17 \mathrm{E}-\varnothing 2 \quad 2.55 \mathrm{E}-\varnothing 3 \quad 7.99 \mathrm{E}-\varnothing 5 \quad 7.8 \varnothing \mathrm{E}-\varnothing 8 \quad 2.38 \mathrm{E}-12 \quad 7.28 \mathrm{E}-17 \quad 2.22 \mathrm{E}-21 \quad 6.75 \mathrm{E}-26 \quad 2 . \varnothing 7 \mathrm{E}-3 \varnothing$

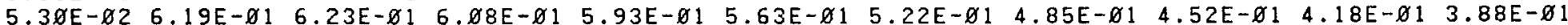
$1.38 E+\varnothing 2,35 E-\varnothing 11.32 E-04 \quad 1.29 E-071.26 E-101.20 E-161.12 E-251.04 E-349.73 E-449.09 E-53 \quad 8.45 E-62$ $1.96 E-011.93 E-011.90 E-011.87 E-011.85 E-011.79 E-011.72 E-011.64 E-011.57 E-011.50 E-011.44 E-01$

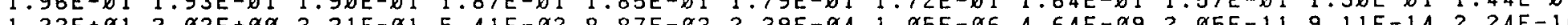

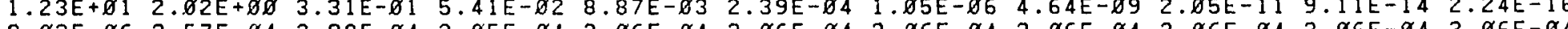
$9.03 \mathrm{E}-062.57 \mathrm{E}-042.98 \mathrm{E}-04 \mathrm{C}$

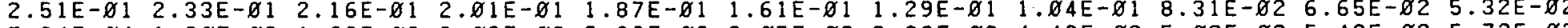
$7.34 \mathrm{E}-\varnothing 4 \quad 1.2 \varnothing \mathrm{E}-\varnothing 3 \quad 1.63 \mathrm{E}-\varnothing 3 \quad 2.02 \mathrm{E}-\varnothing 3 \quad 2.39 \mathrm{E}-\varnothing 3 \quad 3 . \varnothing 5 \mathrm{E}-\varnothing 3 \quad 3.88 \mathrm{E}-\varnothing 3 \quad 4.49 \mathrm{E}-\varnothing 3 \quad 5.03 \mathrm{E}-\varnothing 3 \quad 5.43 \mathrm{E}-\varnothing 3 \quad 5.72 \mathrm{E}-\varnothing 3$

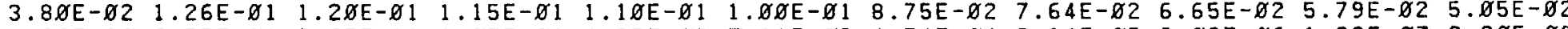

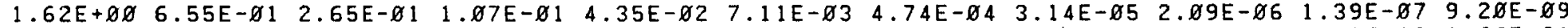

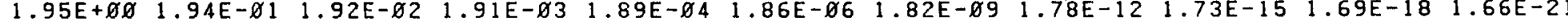
$8.35 E-\varnothing 3 \quad 3.66 E-\varnothing 2 \quad 3.80 E-\varnothing 2 \quad 3.69 E-\varnothing 2 \quad 3.55 E-\varnothing 2 \quad 3.29 E-\varnothing 2 \quad 2.93 E-\varnothing 2 \quad 2.61 E-\varnothing 2 \quad 2.32 E-\varnothing 2 \quad 2 . \varnothing 7 E-\varnothing 2 \quad 1.85 E-\varnothing 2$ $\begin{array}{lllllllllll}1.13 E-\varnothing 4 & 8.12 E-\varnothing 4 & 8 . \varnothing 6 E-\varnothing 4 & 8 . \varnothing 4 E-\varnothing 4 & 8 . \varnothing 1 E-\varnothing 4 & 7.93 E-\varnothing 4 & 7.85 E-\varnothing 4 & 7.74 E-\varnothing 4 & 7.64 E-\varnothing 4 & 7.56 E-\varnothing 4 & 7.45 E-\varnothing 4\end{array}$ 
MR / HR AT $H+12$ HOURS

DEBRIS DECAY FROM 1 TO $3 \not \varnothing$ DAYS

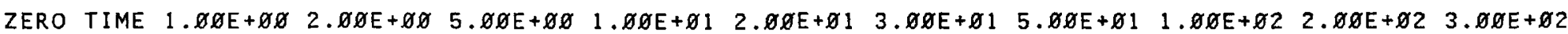

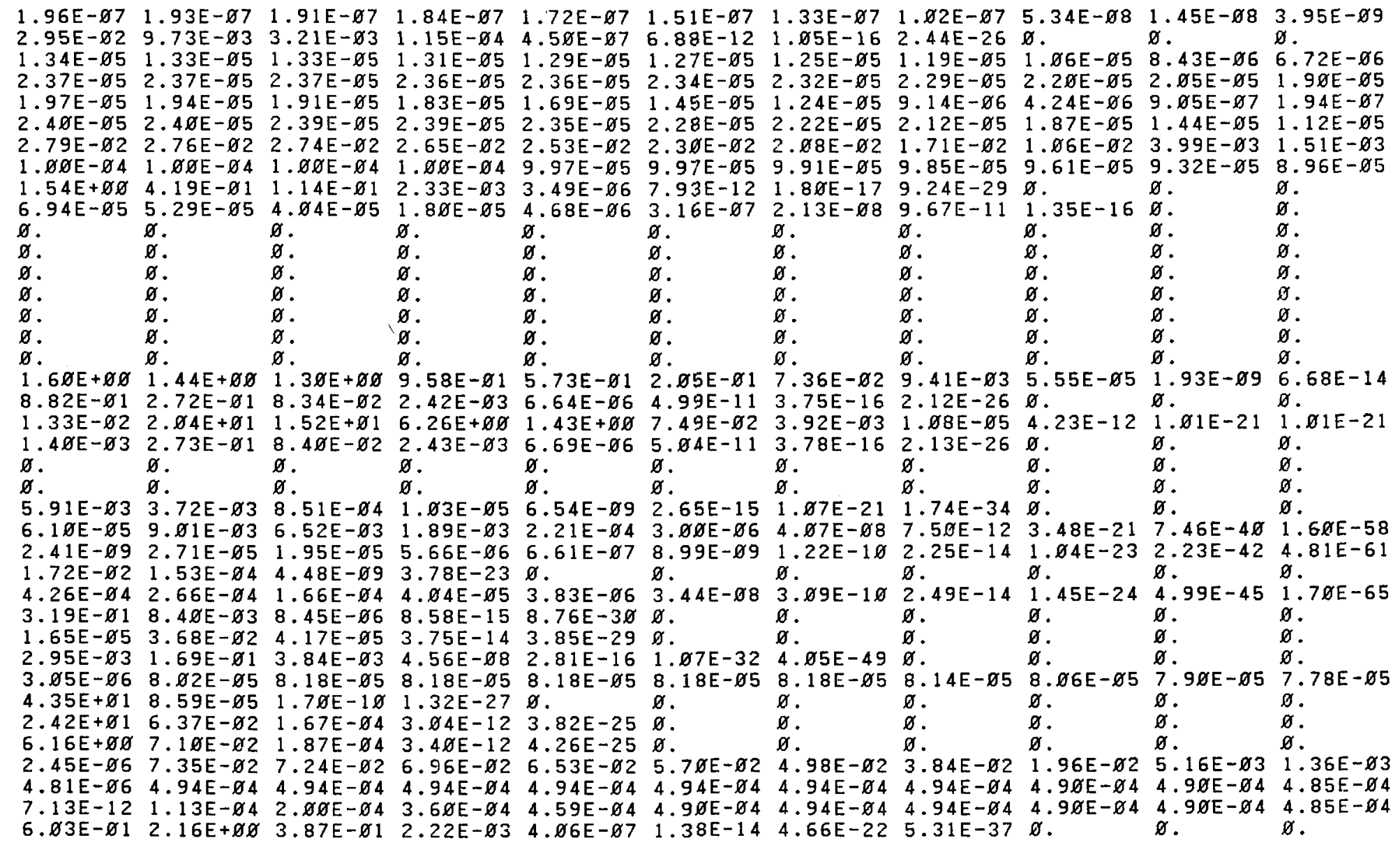


FRACTION OF REFRACTOR IES PRESENT $=\varnothing .5 \varnothing \varnothing$
RELAXATION LENGTH $=\varnothing .16 \mathrm{GM} / \mathrm{SOCM}$

BOMB FRACTION PER SQ. METER $=2.937 E-13$

DEBRIS DECAY FROM 1 TO 300 DAYS

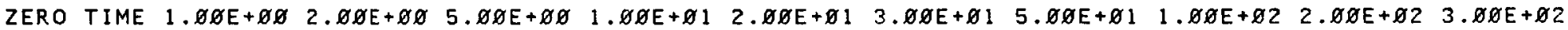

$Y 91 M$

$Y 91$

SR 92

$Y 92$

$Y 93$

ZR 95

NB $95 \mathrm{M}$

NB 95

ZR 97

NB $97 M$

NB $97 \mathrm{M}$

NB 97

$\begin{array}{ll}\text { MO } & 99 \\ \text { TC } & 99 M\end{array}$

RU183

RHIØ3M

RU1ø5

$=$ RH105M

RHIDS

RH 106

RH106

AG IOSM

AG $109 M$

PD111M

PD 111

$A G 111$

$A G 112$

AG 113

CD115M

CD 115

IN $115 \mathrm{M}$

CD 117

IN $117 \mathrm{M}$

IN 117

SN 12

SN123

SN 125

SB 125

SB 126

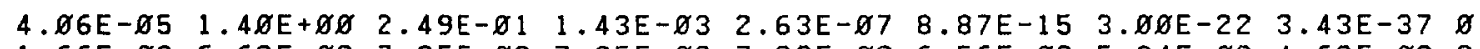

$1.66 \mathrm{E}-08$ 6.63E- 02 7.

(1.82

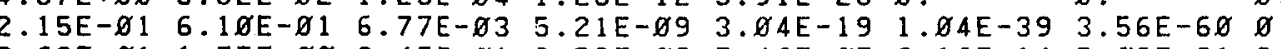

$\begin{array}{lllllll} & & \end{array}$

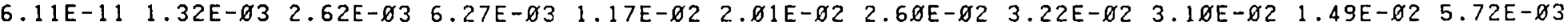

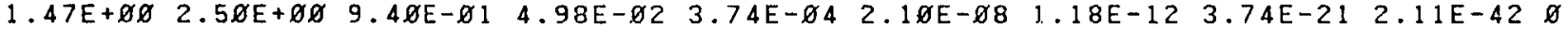

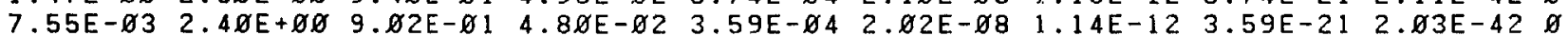

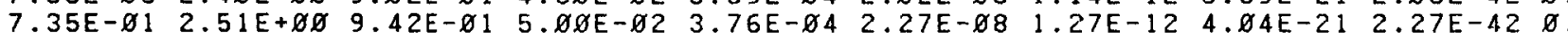

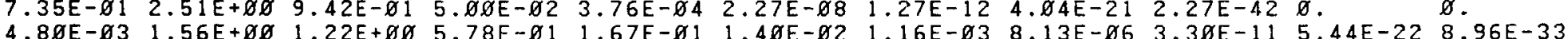

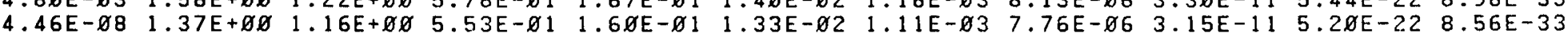
$\begin{array}{lllllllllll}1.28 \mathrm{E}-\varnothing 4 & 2.92 \mathrm{E}-\varnothing 1 & 2.86 \mathrm{E}-\varnothing 1 & 2.72 \mathrm{E}-\varnothing 1 & 2.5 \varnothing \mathrm{E}-\varnothing 1 & 2 . \varnothing 9 \mathrm{E}-\varnothing 1 & 1.76 \mathrm{E}-\varnothing 1 & 1.24 \mathrm{E}-\varnothing 1 & 5.15 \mathrm{E}-\varnothing 2 & 8.95 \mathrm{E}-\varnothing 3 & 1.56 \mathrm{E}-\varnothing 3\end{array}$ $\begin{array}{lllllllllll}8.54 \mathrm{E}-\varnothing 9 & 2.92 \mathrm{E}-\varnothing 1 & 2.87 \mathrm{E}-\varnothing 1 & 2.73 \mathrm{E}-\varnothing 1 & 2.5 \varnothing \mathrm{E}-\varnothing 1 & 2 . \varnothing 9 \mathrm{E}-\varnothing 1 & 1.76 \mathrm{E}-\varnothing 1 & 1.24 \mathrm{E}-\varnothing 1 & 5.16 \mathrm{E}-\varnothing 2 & 8.95 \mathrm{E}-\varnothing 3 & 1.56 \mathrm{E}-\varnothing 3\end{array}$ $\begin{array}{lllllll}1.11 \mathrm{E}+\varnothing \varnothing 0 & 1 . \varnothing 6 \mathrm{E}+\varnothing \varnothing \varnothing 2.49 \mathrm{E}-\varnothing 2 & 3.28 \mathrm{E}-\varnothing 7 & 2.4 \varnothing \mathrm{E}-15 & 1.28 \mathrm{E}-31 & 6.85 \mathrm{E}-48 & \varnothing\end{array}$ $\begin{array}{llllllll}7.29 E-\varnothing 3 & 1 . \varnothing 6 E+\varnothing \varnothing \varnothing & 2.5 \varnothing E-\varnothing 2 & 3.29 E-\varnothing 7 & 2.4 \varnothing E-15 & 1.29 E-31 & 6.87 E-48 & \varnothing\end{array}$ Ø.

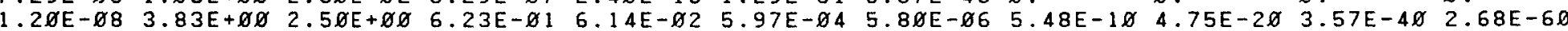

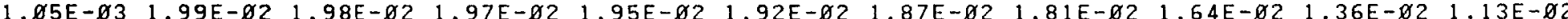

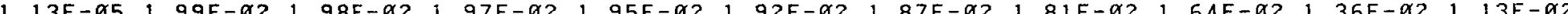
$1.86 E-02$ - $73 E-01$ .

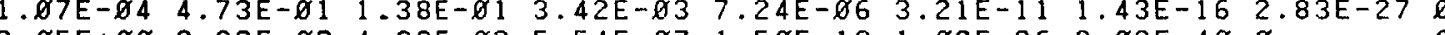

$2.05 \mathrm{E}+\varnothing \varnothing 09.93 \mathrm{E}-\varnothing 2 \quad 4.83 \mathrm{E}-\varnothing 3 \quad 5.54 \mathrm{E}-\varnothing 7 \quad 1.5 \varnothing \mathrm{E}-13 \quad 1.09 \mathrm{E}-26 \quad 8.02 \mathrm{E}-4 \varnothing \quad \varnothing$

$\begin{array}{llllllll}7.29 \mathrm{E}-\emptyset 1 & 8.02 \mathrm{E}-\varnothing 2 & 3.88 \mathrm{E}-\varnothing 3 & 4.46 \mathrm{E}-\varnothing 7 & 1.21 \mathrm{E}-13 & 8.79 \mathrm{E}-27 & 6.44 \mathrm{E}-4.0 & \varnothing .\end{array}$

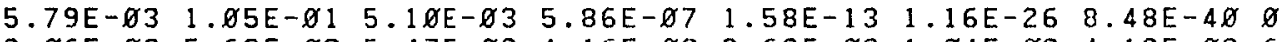

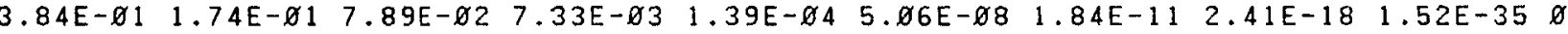

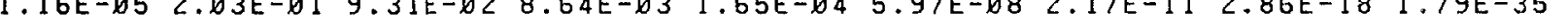

$2.91 E-\varnothing 3 \quad 3.07 E-\varnothing 2 \quad 1.33 E-\varnothing 3 \quad 1.08 E-\varnothing 7 \quad 1.65 E-14 \quad 3.87 E-28 \quad 9.03 E-42 \quad 0$.

$\varnothing$.

$\varnothing$. $\varnothing$

.

$\varnothing$. 0 S.

$\begin{array}{lllllllllll}.74 E-\varnothing 9 & 1.95 E-\varnothing 4 & 1.92 E-\varnothing 4 & 1.83 E-\varnothing 4 & 1.69 E-\varnothing 4 & 1.43 E-\varnothing 4 & 1.22 E-\varnothing 4 & 8.84 E-\varnothing 5 & 3.95 E-\varnothing 5 & 7.86 E-\varnothing 6 & 1.57 E-\varnothing 6\end{array}$ .

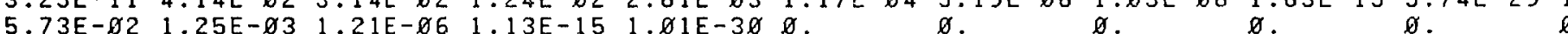

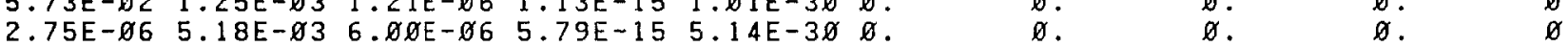

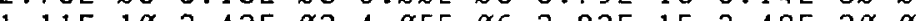
$1.11 \mathrm{E}-1 \varnothing \quad 3.42 \mathrm{E}-\varnothing 3 \quad 4.05 \mathrm{E}-\varnothing 6 \quad 3.92 \mathrm{E}-15 \quad 3.48 \mathrm{E}-3 \varnothing \quad \varnothing$ Ø.

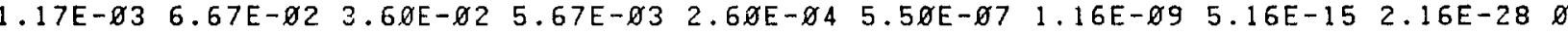

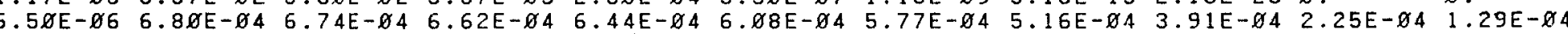

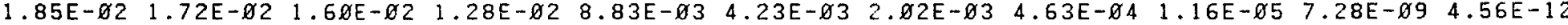

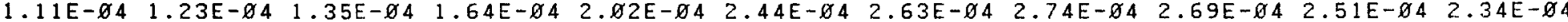

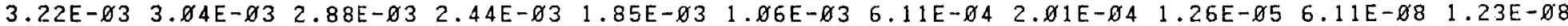


DEBRIS DECAY FROM 1 TO 300 DAYS

SN 127

SB 127

TE $127 M$

TE 127

SB 128

SB 129

TE $129 M$

TE 129

I 130

TE $131 \mathrm{M}$

XE131

XEI31M

TE 132
I 132

I 133

N XE133M

$X E 133$

$I 135$
$X E 135 M$

XE135M

$\operatorname{cs} 136$

$\operatorname{CS} 137$

BA1 $37 \mathrm{M}$

BA 139

BA140

LA 140

LAI 41

CE 141

LA 142

CE 143

PR 143

CE 144

PR 144

PR 145

ND 147

PM1 47

ND 149

PDI 149

PM150
ZERO TIME $1 . \varnothing \varnothing E+\varnothing \varnothing \varnothing 2 . \varnothing \varnothing E+\varnothing \varnothing \quad 5 . \varnothing \varnothing E+\varnothing \varnothing \quad 1 . \varnothing \varnothing E+\varnothing 1 \quad 2 . \varnothing \varnothing E+\varnothing 1 \quad 3 . \varnothing \varnothing E+\varnothing 1 \quad 5 . \varnothing \varnothing E+\varnothing 1 \quad 1 . \varnothing \varnothing E+\varnothing 2 \quad 2 . \varnothing \varnothing E+\varnothing 2 \quad 3 . \varnothing \varnothing E+\varnothing 2$ $\begin{array}{llllll}3.28 E+\varnothing 0 & 1.19 E-\not 3 & 4.32 E-\varnothing 7 & 2.06 E-17 & 1.29 E-34 & \varnothing .\end{array}$

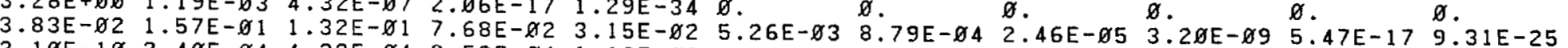

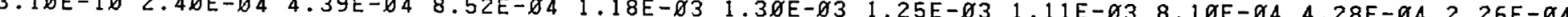

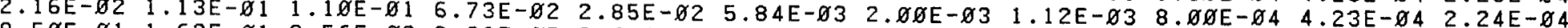

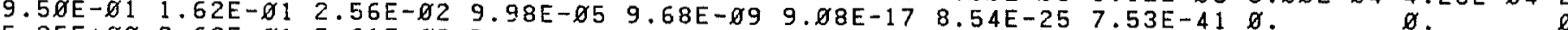

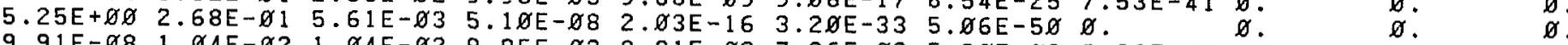

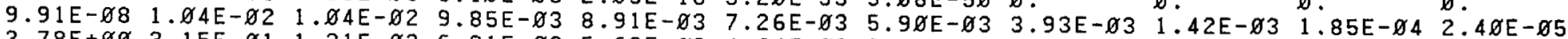

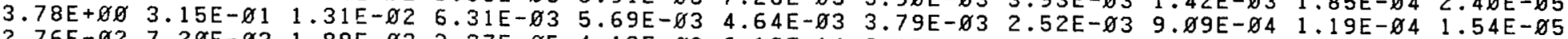

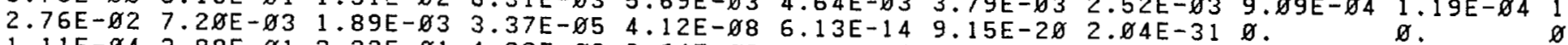

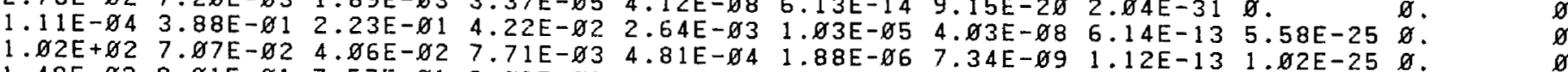

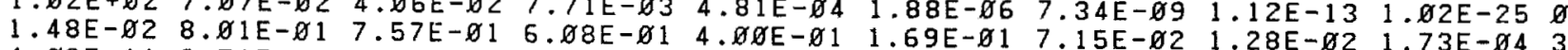

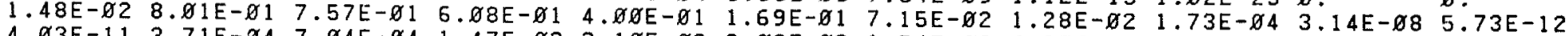
1.00

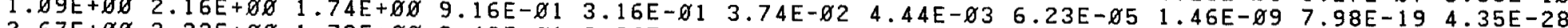

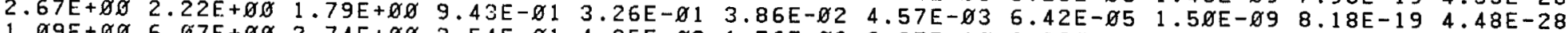

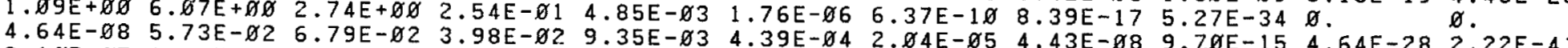

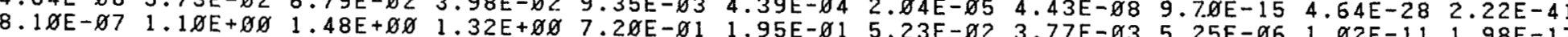

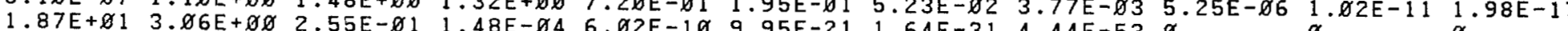

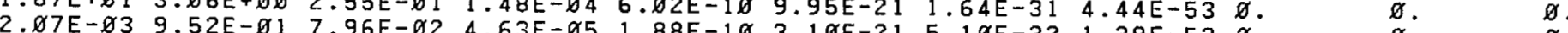

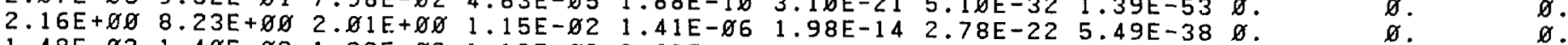
$1.48 \mathrm{E}-\varnothing 2 \quad 1.4 \varnothing \mathrm{E}-\varnothing 2$ 1.33E-Ø2 $1.13 \mathrm{E}-\varnothing 2 \quad 8.69 \mathrm{E}-\varnothing 3 \quad 5.1 \varnothing \mathrm{E}-\varnothing 3 \quad 3 . \varnothing \varnothing \mathrm{E}-\varnothing 3 \quad 1 . \varnothing 3 \mathrm{E}-\varnothing 3 \quad 7.16 \mathrm{E}-\varnothing 5 \quad 3.45 \mathrm{E}-\varnothing 7 \quad 1.67 \mathrm{E}-\varnothing 9$ $9.36 \mathrm{E}-\varnothing 5 \quad 1.01 \mathrm{E}-\varnothing 3 \quad 1.01 \mathrm{E}-\varnothing 3 \quad 1 . \varnothing 1 \mathrm{E}-\varnothing 3 \quad 1 . \varnothing 1 \mathrm{E}-\varnothing 3 \quad 1 . \varnothing 1 \mathrm{E}-\varnothing 3 \quad 1 . \varnothing 1 \mathrm{E}-\varnothing 3 \quad 1 . \varnothing 1 \mathrm{E}-\varnothing 3 \quad 1 . \varnothing \varnothing \mathrm{E}-\varnothing 3 \quad 9.97 \mathrm{E}-\varnothing 4 \quad 9.92 \mathrm{E}-\varnothing 4$

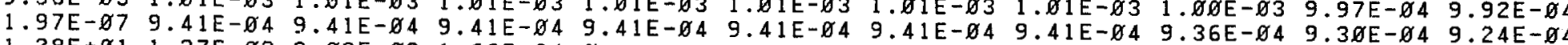

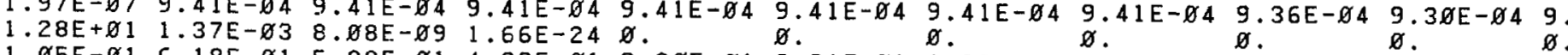

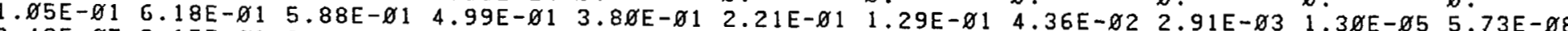

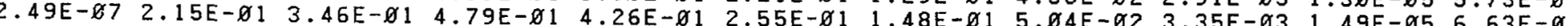

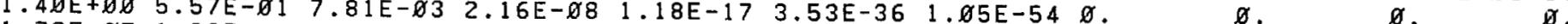

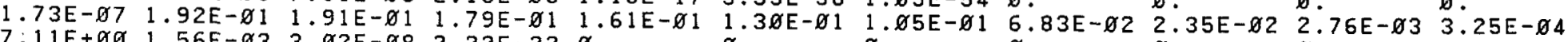

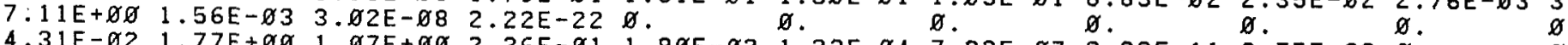

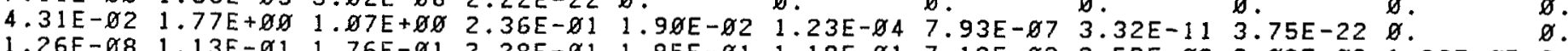

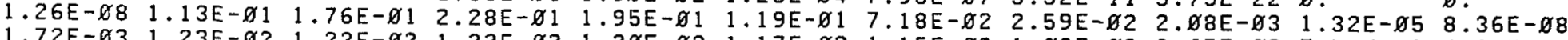

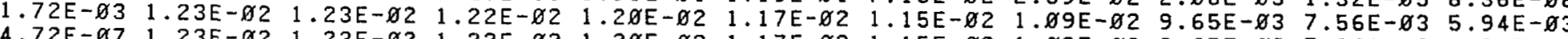

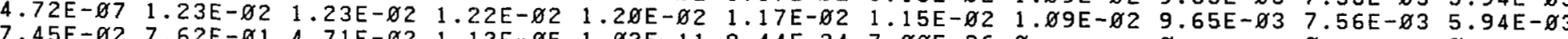

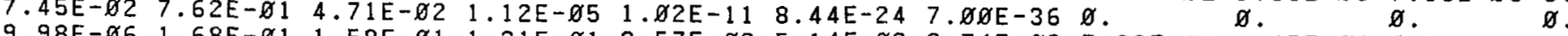

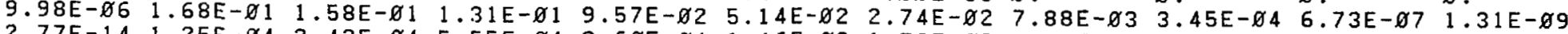

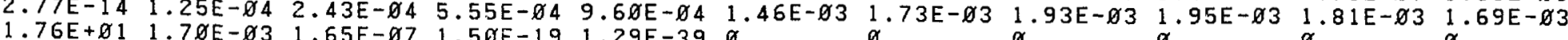

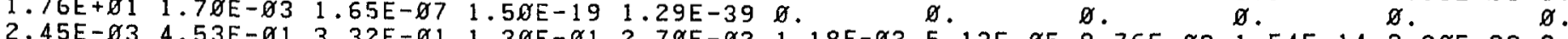

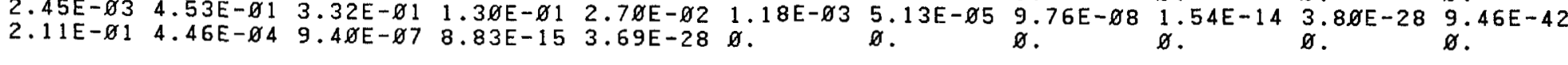


TR INITY

MICROCURIES/SO METER

FRACTION OF REFRACTORIES PRESENT $=\varnothing .5 \varnothing \varnothing$

RELAXATION LENGTH $=\varnothing .16 \mathrm{GM} / \mathrm{SQCM}$

BOMB FRACTION PER SQ. METER $=2.937 E-13$

DEBRIS DECAY FROM 1 TO 300 DAYS

PM151

SM153

SM 156

EU155

EU156

EU157

GD 159
TB 161

TOTAL

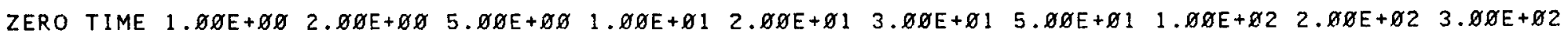

$\begin{array}{llllllllll}5.3 \varnothing \mathrm{E}-\varnothing 2 & 3.61 \mathrm{E}-\varnothing 1 & 1.99 \mathrm{E}-\varnothing 1 & 3.35 \mathrm{E}-\varnothing 2 & 1.72 \mathrm{E}-\varnothing 3 & 4.52 \mathrm{E}-\varnothing 6 & 1.19 \mathrm{E}-\varnothing 8 & 8.21 \mathrm{E}-14 & 1.03 \mathrm{E}-26 \quad 0 .\end{array}$

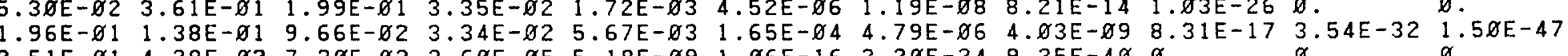

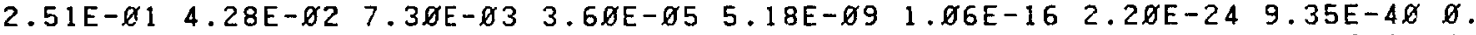

0 .

$9 . \varnothing 3 \mathrm{E}-\varnothing 6 \quad 3 . \varnothing 6 \mathrm{E}-\varnothing 4 \quad 3 . \varnothing 5 \mathrm{E}-\varnothing 4 \quad 3 . \varnothing 5 \mathrm{E}-\varnothing 4 \quad 3 . \varnothing 5 \mathrm{E}-\varnothing 4 \quad 3 . \varnothing 3 \mathrm{E}-\varnothing 4 \quad 3 . \varnothing 2 \mathrm{E}-\varnothing 44 \quad 3 . \varnothing \varnothing \mathrm{E}-\varnothing 4 \quad 2.94 \mathrm{E}-\varnothing 42.82 \mathrm{E}-\varnothing 4 \quad 2.71 \mathrm{E}-\varnothing 4$

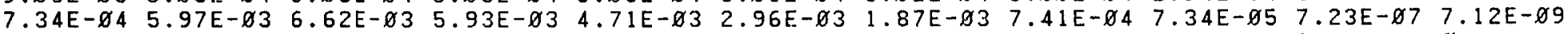

$3.8 \varnothing \mathrm{E}-\varnothing 2 \quad 4.4 \varnothing \mathrm{E}-\varnothing 2 \quad 1.48 \mathrm{E}-\varnothing 2 \quad 5.55 \mathrm{E}-\varnothing 4 \quad 2.33 \mathrm{E}-\varnothing 6 \quad 4.11 \mathrm{E}-11 \quad 7.27 \mathrm{E}-16 \quad 2.26 \mathrm{E}-25 \quad \varnothing$

$\varnothing$.

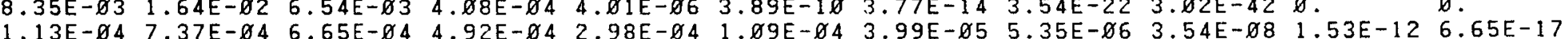

$2.72 E+\varnothing 2 \quad 7.98 E+\varnothing 1 \quad 4.17 E+\varnothing 1 \quad 1.64 E+\varnothing 1 \quad 6.43 E+\varnothing \varnothing \quad 2.29 E+\varnothing \varnothing \quad 1.34 E+\varnothing \varnothing \quad 7.22 E-\varnothing 1 \quad 3 . \varnothing 9 E-\varnothing 1 \quad 1.1 \varnothing E-\varnothing 1 \quad 5.76 E-\varnothing 2$ 
FRACTION OF REFRACTORIES PRESENT $=\varnothing .500$

RELAXATION LENGTH $=\varnothing .16 \mathrm{GM} / \mathrm{SQCM}$

BOMB FRACTION PER SQ. METER $=2.937 \mathrm{E}-13$

DEBRIS DECAY FROM 1 TO $5 \varnothing$ YEARS

ZERO TIME $1 . \varnothing \varnothing E+\varnothing \varnothing \quad 1.5 \varnothing E+\varnothing \varnothing \quad 2 . \varnothing \varnothing E+\varnothing \varnothing \quad 3.5 \varnothing E+\varnothing \varnothing \quad 5 . \varnothing \varnothing E+\varnothing \varnothing \quad 7 . \varnothing \varnothing E+\varnothing \varnothing \quad 1 . \varnothing \varnothing E+\varnothing 1 \quad 2 . \varnothing \varnothing E+\varnothing 1 \quad 3.5 \varnothing E+\varnothing 1 \quad 5 . \varnothing \varnothing E+\varnothing 1$

*BE 7

*MN 54

*FE 59

*CO 57

*CO 58

*CO 68

$W 181$

W1 85

W1 88

AM2 41

CM2 42

CM242

KR 85

SR 89

SR 90

$Y 90$

ZR 95

NB $95 \mathrm{M}$

NB 95

RU1Ø3

RH1Ø3M

RU106

RH 106

SN123

SB 125

TE $125 \mathrm{M}$

TE $127 \mathrm{M}$

TE 127

CS 137

BA $137 \mathrm{M}$

CE 141

CE 144

TOTAL

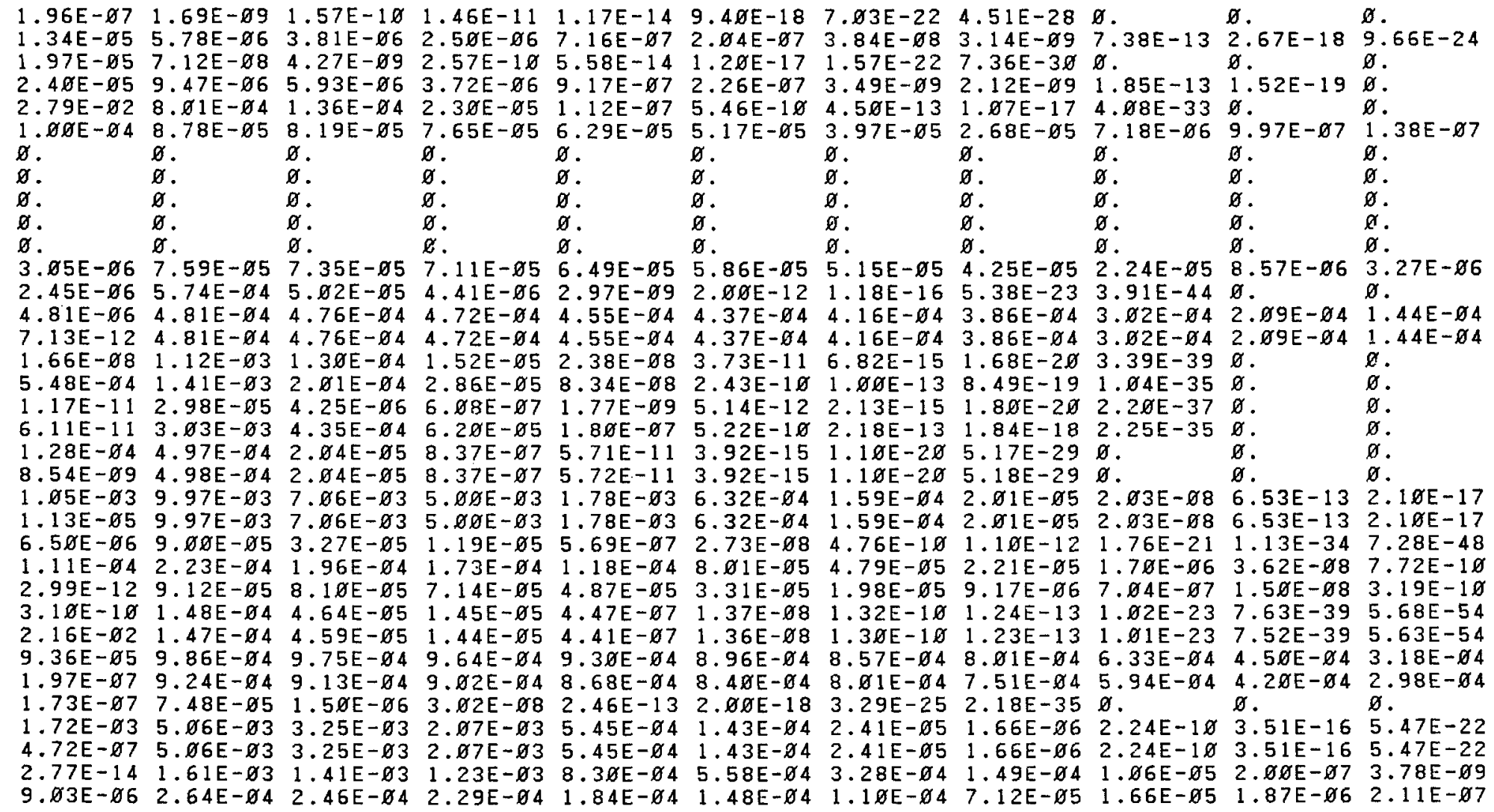

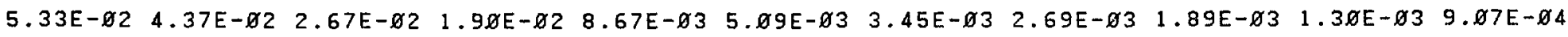




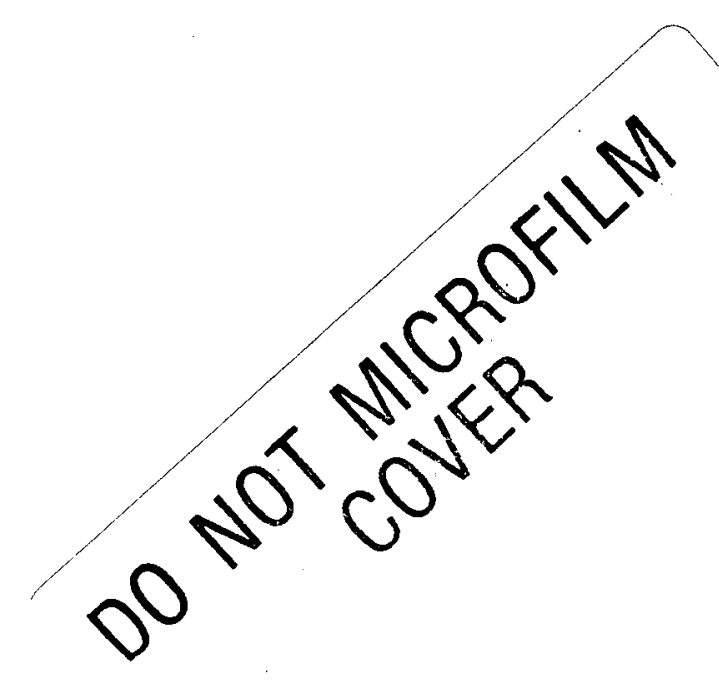

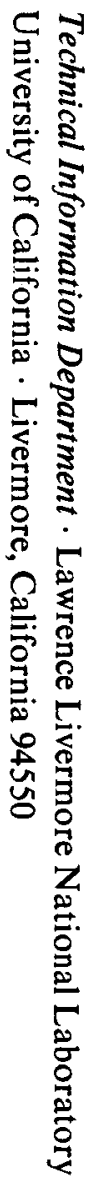

